\title{
Improved Fractional Subequation Method and Exact Solutions to Fractional Partial Differential Equations
}

\author{
Jun Jiang, ${ }^{1}$ Yuqiang Feng $\mathbb{D}^{1,2}$ and Shougui $\mathrm{Li}^{2}$ \\ ${ }^{1}$ School of Science, Wuhan University of Science and Technology, Wuhan, 430065 Hubei, China \\ ${ }^{2}$ Hubei Province Key Laboratory of Systems Science in Metallurgical Process, Wuhan, 430065 Hubei, China \\ Correspondence should be addressed to Yuqiang Feng; yqfeng6@126.com
}

Received 2 February 2020; Accepted 13 April 2020; Published 17 May 2020

Academic Editor: Xinguang Zhang

Copyright ( 2020 Jun Jiang et al. This is an open access article distributed under the Creative Commons Attribution License, which permits unrestricted use, distribution, and reproduction in any medium, provided the original work is properly cited.

In this paper, the improved fractional subequation method is applied to establish the exact solutions for some nonlinear fractional partial differential equations. Solutions to the generalized time fractional biological population model, the generalized time fractional compound $\mathrm{KdV}$-Burgers equation, the space-time fractional regularized long-wave equation, and the $(3+1)$-spacetime fractional Zakharov-Kuznetsov equation are obtained, respectively.

\section{Introduction}

Fractional differential equations are widely used to describe lots of important phenomena and dynamic processes in physics, engineering, electromagnetics, acoustics, viscoelasticity electrochemistry, material science, stochastic dynamical system, plasma physics, controlled thermonuclear fusion, nonlinear control theory, image processing, nonlinear biological systems and astrophysics, etc. [1-7]. In order to find the solutions of fractional differential equations, many powerful and efficient methods have been introduced and developed, such as Darboux transformations [8], the hyperbolic function method [9], the variational iteration method $[10,11]$, the autofinite element method $[12,13]$, the auxiliary equation method [14], the finite difference method $[15,16]$, the Adomian decomposition method [17, 18], the homogenous balance method [19], Hirotas bilinear method [20], the homotopy analysis method [21, 22], $\left(G^{\prime} / G\right)$-expansion method $[23,24]$, the subequation method $[25,26]$, the first integral method $[27,28]$, the improved fractional subequation method $[29,30]$, the extended Jacobi elliptic function expansion method [31], the generalized Kudryashov method [32], the exponential rational function method [33], the expfunction method $[34,35]$, the multiple exp-function method [36], the extended simple equation method [37].

In order to deal with nondifferentiable functions, Jumarie [38] has proposed a modification of the Riemann-Liouville definition which appears to provide a framework for a fractional calculus which is quite parallel to the classical calculus.

Jumarie's modified Riemann-Liouville derivative of order $\alpha$ for a function $f$ is defined as follows:

$$
D_{x}^{\alpha} f(x)= \begin{cases}\frac{1}{\Gamma(1-\alpha)} \int_{0}^{x}(x-\xi)^{-\alpha-1}[f(\xi)-f(0)] d \xi, & \alpha<0, \\ \frac{1}{\Gamma(1-\alpha)} \frac{d}{d x} \int_{0}^{x}(x-\xi)^{-\alpha}[f(\xi)-f(0)] d \xi, & 0<\alpha<1, \\ {\left[f^{(n)}(x)\right]^{(\alpha-n)},} & n \leq \alpha<n+1, n \geq 1 .\end{cases}
$$


Some useful properties of modified Riemann-Liouville derivative are given below:

$$
\begin{gathered}
D_{x}^{\alpha} x^{\gamma}=\frac{\Gamma(\gamma+1)}{\Gamma(\gamma+1-\alpha)} x^{\gamma-\alpha}, \gamma>0, \\
D_{x}^{\alpha}[f(x) g(x)]=g(x) D_{x}^{\alpha} f(x)+f(x) D_{x}^{\alpha} g(x), \\
D_{x}^{\alpha} f[g(x)]=f_{g}^{\prime}[g(x)] D_{x}^{\alpha} g(x)=D_{g}^{\alpha} f[g(x)]\left[g^{\prime}(x)\right]^{\alpha},
\end{gathered}
$$

which holds for nondifferentiable functions. Equations (2), (3), (4) which are important tools for fractional calculus. Based on these merits, the modified Riemann-Liouville derivative was successfully applied to the probability calculus, fractional Laplace problems, and fractional variational calculus.

In this paper, we aim to find new exact solutions of some important partial fractional differential equations under Jumarie's definition by improved fractional subequation method.

In what follows, we introduce the aforementioned fractional partial differential equations. They are the generalized time fractional biological population model, the generalized time fractional compound $\mathrm{KdV}$-Burgers equation, the spacetime fractional regularized long-wave equation, and the $(3+1)$-space-time fractional Zakharov-Kuznetsov equation.

Suppose time $t>0, D_{t}^{\alpha} u$ is time modified RiemannLiouville derivative of order $\alpha$ for a function $u, 0<\alpha \leq 1$, the parameters $k_{1}, k_{2}, \cdots, k_{7}$ are any real constants.

The generalized time fractional biological population model is given by

$D_{t}^{\alpha} u+k_{1}\left(u^{2}\right)_{x x}+k_{2}\left(u^{2}\right)_{y y}+k_{3} u_{x}+k_{4} u_{y}+k_{5} u^{2}+k_{6} u+k_{7}=0$,

where $u=u(x, y, t)$ is an unknown function.

When $k_{1}=-1, k_{2}=-1, k_{3}=k_{4}=k_{6}=0, k_{5}=-h \neq 0, k_{7}=$ $r h \neq 0$, Equation (5) is the time fractional biological population model [39]:

$$
D_{t}^{\alpha} u=\left(u^{2}\right)_{x x}+\left(u^{2}\right)_{y y}+h\left(u^{2}-r\right),
$$

where $u=u(x, y, t)$ denotes the population density and $h$ $\left(u^{2}-r\right)$ represents the amount of population due to death and birth. Moreover, $h\left(u^{2}-r\right)$ leads to Verhulst law. Equation (5) has an important role to understand the dynamic process of population changes, and it is also an assistant to achieve precision about it.

The generalized time fractional compound KdV-Burgers equation is given by

$$
D_{t}^{\alpha} u+k_{1} u u_{x}+k_{2} u^{2} u_{x}+k_{3} u_{x x}+k_{4} u_{x x x}=0 \text {, }
$$

where $u=u(x, t)$ is an unknown function.

When $k_{1}=k_{3}=0$, Equation (7) becomes the time fractional $\mathrm{mKdV}$ equation

$$
D_{t}^{\alpha} u+k_{2} u^{2} u_{x}+k_{4} u_{x x x}=0
$$

when $k_{1}=k_{3}=0$ Equation (7) becomes the time fractional $\mathrm{KdV}$ equation

$$
D_{t}^{\alpha} u+k_{2} u^{2} u_{x}+k_{3} u_{x x}+k_{4} u_{x x x}=0
$$

when $k_{2}=k_{4}=0$, Equation (7) becomes the time fractional Burgers equation [40]

$$
D_{t}^{\alpha} u+k_{1} u u_{x}+k_{3} u_{x x}=0
$$

when $k_{1}=0$, Equation (7) becomes the time fractional $\mathrm{mKdV}$-Burgers equation

$$
D_{t}^{\alpha} u+k_{2} u^{2} u_{x}+k_{3} u_{x x}+k_{4} u_{x x x}=0
$$

when $k_{2}=0$, Equation (7) becomes the time fractional $\mathrm{KdV}$ Burgers equation

$$
D_{t}^{\alpha} u+k_{1} u u_{x}+k_{3} u_{x x}+k_{4} u_{x x x}=0 .
$$

The space-time fractional regularized long-wave equation is given by [41]:

$$
D_{t}^{\alpha} u+k_{1} D_{x}^{\alpha} u+k_{2} u D_{x}^{\alpha} u+k_{3} D_{t}^{\alpha} D_{x}^{2} \alpha u=0
$$

where $u=u(x, t)$ is an unknown function, $D_{x}^{\alpha} u$ is the modified Riemann-Liouville derivative of order $\alpha$ for a function $u$, and $D_{x}^{2 \alpha} u=D_{x}^{\alpha}\left(D_{x}^{\alpha} u\right)$.

The regularized long-wave equation, which describes approximately the unidirectional propagation of long waves in certain nonlinear dispersive systems, was proposed by Benjamin et al. in 1972. The regularized long-wave equation is considered an alternative to the $\mathrm{KdV}$ equation, which is modeled to govern a large number of physical phenomena such as shallow waters and plasma waves.

The $(3+1)$-space-time fractional Zakharov-Kuznetsov equation given by [41].

$$
D_{t}^{?} u+k_{1} u D_{x}^{\alpha} u+k_{2} D_{x}^{3 \alpha} u+k_{3} D_{x}^{\alpha} D_{y}^{2 \alpha} u+k_{4} D_{x}^{\alpha} D_{z}^{2 \alpha} u=0,
$$

where $u=u(x, y, z, t)$ is an unknown function; $D_{x}^{\alpha} u, D_{y}^{\alpha} u$, and $D_{z}^{\alpha} u$ are the modified Riemann-Liouville derivatives of the function $u ; D_{x}^{2 \alpha} u=D_{x}^{\alpha}\left(D_{x}^{\alpha} u\right) ; D_{y}^{2 \alpha} u=D_{y}^{\alpha}\left(D_{y}^{\alpha} u\right) ; D_{z}^{2 \alpha} u=D_{z}^{\alpha}$ $\left(D_{z}^{\alpha} u\right)$; and $D_{x}^{3 \alpha} u=D_{x}^{\alpha}\left(D_{x}^{\alpha}\left(D_{x}^{\alpha} u\right)\right)$.

The Zakharov-Kuznetsov equation was first derived for analysing weakly nonlinear ion acoustic waves in heavily magnetized lossless plasma and geophysical flows in two dimensions. The $\mathrm{ZK}$ equation is one of the two wellestablished canonical two-dimensional extensions of the $\mathrm{KdV}$ equation. The $\mathrm{ZK}$ equation governs the behavior of weakly nonlinear ionacoustic waves in a plasma comprising cold ions and hot isothermal electrons in the presence of a uniform magnetic field.

Motivated by the above results, in this paper, we use the improved subequation method to find new exact solutions of the generalized time fractional biological population model, the generalized time fractional compound KdV-Burgers equation, the space-time fractional regularized long-wave equation, 
and the $(3+1)$-space-time fractional Zakharov-Kuznetsov equation, respectively.

\section{A Brief Description of the Improved Fractional Subequation Method}

In this section, basic steps of the improved subequation method [42] are presented.

Consider the following nonlinear fractional differential equation,

$$
\begin{aligned}
& P\left(u, D_{t}^{\alpha} u, D_{x_{1}}^{\alpha} u, D_{x_{1}}^{\alpha} u^{2}, \cdots, D_{x_{m}}^{\alpha} u, D_{x_{1}}^{\alpha} u^{2},\right. \\
& \left.D_{x_{2}}^{\alpha} u^{2}, \cdots, D_{x_{m}}^{\alpha} u^{2}, \cdots, D_{x_{1}}^{2 \alpha} u, D_{x_{m}}^{2 \alpha} u, \cdots\right)=0,
\end{aligned}
$$

where $t, x_{1}, x_{2}, \cdots, x_{m}$ are independent variables, $u\left(t, x_{1}, x_{2}\right.$, $\left.\cdots, x_{m}\right)$ is an unknown function, and $P$ is a polynomial of $u$, $u^{2}, \cdots$ and their partial fractional derivatives. Also, $D^{\alpha}(\cdot)$ symbolizes the modified Riemann-Liouville fractional derivative.

Step 1. First of all, using a suitable fractional complex transform,

$$
u\left(t, x_{1}, x_{2}, \cdots, x_{m}\right)=u(\xi), \xi\left(t, x_{1}, x_{2}, \cdots \mid, x_{m}\right),
$$

Equation (15) converts into nonlinear ordinary differential equation given below:

$$
Q\left(u, u^{\prime}, u^{\prime \prime}, u^{\prime \prime \prime}, \cdots\right)=0
$$

where $u^{\prime}, u^{\prime \prime}, u^{\prime \prime \prime}, \cdots$ denotes the derivations with respect to $\xi$.

We used to utilize real transformation, but actually, complex transformations are more useful. They make some equations easier to simplify.

Step 2. Suppose that the solution of ordinary differential equation (17) is

$$
u=\sum_{i=n}^{-1} a_{i} \varphi^{i}+a_{0}+\sum_{i=n}^{n} a_{i} \varphi^{i}
$$

where constants $a_{i}(i=-n, \cdots,-1,1, \cdots, n)$ are going to be determined. Here, $n$ is a positive integer, and it is obtained using the homogeneous balance of the highest order derivative and the nonlinear term seen in Equation (17).

$\varphi=\varphi(\xi)$ is the solution of the Riccati equation

$$
\varphi^{\prime}=\sigma+\varphi^{2},
$$

where $\sigma$ is a constant, and the solutions of Equation (19) are obtained by Zhang et al. [34] as follows:

$$
\varphi(\xi)= \begin{cases}-\sqrt{-\sigma} \tanh (\mid-\sigma \xi, & \sigma<0, \\ -\sqrt{-\sigma} \operatorname{coth}(\mid-\sigma \xi, & \sigma<0, \\ \sqrt{\sigma} \tan (\mid \bar{\xi}), & \sigma>0, \\ -\sqrt{\sigma} \cot (\mid \bar{\xi}), & \sigma>0, \\ -\frac{\Gamma(1+\alpha)}{\xi^{\alpha}+\omega}, & \sigma=0, \omega=\text { const }\end{cases}
$$

In the previous literatures, $u=a_{0}+\sum_{i=1}^{n} a_{i} \varphi^{i}$ was considered. In this paper, we assume $u=\sum_{i=-n}^{-1} a_{i} \varphi^{i}+a_{0}+\sum_{i=1}^{n} a_{i} \varphi^{i}$, and we can get a solution that has both hyperbolic tangent function and hyperbolic cotangent function or both tangent function and cotangent function.

Step 3. Putting Equation (18) along with Equation (19) into Equation (17), we obtain a new polynomial in terms of $\varphi$. Then, all the coefficients of powers of $\varphi^{k}(k=0,1,2$, $\cdots,-1,-2, \cdots)$ are set equal to zero, we get a system of algebraic equations.

Step 4. Finally, the system of algebraic equations is obtained in the previous step for $a_{i}(-n \leq i \leq n)$, and $\sigma$ is solved by the Maple package. By substituting the newly obtained values into Equation (20), we get the exact solutions for the nonlinear fractional differential equation (15).

Applying a suitable fractional complex transform of the improved fractional subequation method and the chain rule, nonlinear fractional differential equations with the modified Riemann-Liouville derivative can be converted into nonlinear ordinary differential equations. Then, using the solutions of a Riccati equation, we can find exact analytical solutions expressed by triangle functions, hyperbolic functions, or power functions.

\section{Applications of the Improved Fractional Subequation Method}

In this section, the improved fractional subequation method is utilized to solve some nonlinear fractional differential equations introduced in Section 1.

3.1. The Generalized Time Fractional Biological Population Model. The generalized time fractional biological population model is given by

$$
\begin{aligned}
& D_{t}^{\alpha} u+k_{1}\left(u^{2}\right)_{x x}+k_{2}\left(u^{2}\right)_{y y}+k_{3} u_{x}+k_{4} u_{y} \\
& \quad+k_{5} u^{2}+k_{6} u+k_{7}=0,
\end{aligned}
$$

where $u=u(x, y, t)$ is an unknown function.

We considered two cases.

Case 1. When $k_{1} k_{2}>0$, let

$$
u(x, y, t)=u(\xi), \xi=a x+i a \sqrt{\frac{k_{1}}{k_{2}}} y+\frac{b t^{\alpha}}{\Gamma(1+\alpha)}, a \neq 0,
$$


then Equation (21) is reduced to the ordinary differential equation as follows:

$$
\left\{\begin{array}{l}
a k_{4} \sqrt{\frac{k_{1}}{k_{2}}} u^{\prime}=0, \\
\left(a k_{3}+b\right) u^{\prime}+k_{5} u^{2}+k_{6} u+k_{7}=0 .
\end{array}\right.
$$

When $k_{4} \neq 0$, Equation (23) has only constant solutions.

When $k_{4}=0$, Equation (23) has solutions in the form of (18). $u^{\prime}$ is obtained from the homogeneous balance between the highest order derivative and the nonlinear term $u^{2}$. We obtain the solution of Equation (21) as follows:

$$
u(\xi)=a-1 \varphi^{-1}+a_{0}+a_{1} \varphi,
$$

Substituting Equation (24) together with its necessary derivatives into Equation (21), the algebraic equation is arranged according to the powers of the function $\varphi^{k}(\xi)$. Then, the following coefficients are obtained:

$$
\begin{aligned}
\varphi^{-2}: & -k_{3} \sigma a_{-1}-b \sigma a_{-1}+k_{5} a_{-1}^{2}, \\
\varphi^{-1}: & 2 k_{5} a_{-1} a_{0}+a_{-1} k_{6}, \\
\varphi^{0}: & -a k_{3} a_{-1}-b a_{-1}+a k_{3} \sigma a_{1}+b \sigma a_{1} \\
& +k_{5} a_{0}^{2}+2 k_{5} a_{-1} a_{1}+k_{6} a_{0}+k_{7}, \\
\varphi^{1}: & 2 k_{5} a_{0} a_{1}+k_{6} a_{1}, \\
\varphi^{2}: & a k_{3} a_{1}+b a_{1}+k_{5} a_{1}^{2} .
\end{aligned}
$$

Let the coefficients be zero. By solving the set of equations given above for $a_{-1}, a_{0}, a_{1}, a, b$, and $\sigma$, we obtain solution sets as follows:

Set 1

$$
\begin{aligned}
a_{-1} & =0, \\
a_{0} & =-\frac{k_{6}}{2 k_{5}}, \\
a_{1} & =a_{1}, \\
a & =a, \\
b & =-a k_{3}-a_{1} k_{5}, \\
\sigma & =\frac{\lambda}{4 a_{1}^{2} k_{5}^{2}} .
\end{aligned}
$$

Set 2

$$
\begin{aligned}
a_{-1} & =a_{-1}, \\
a_{0} & =-\frac{k_{6}}{2 k_{5}}, \\
a_{1} & =\frac{-\lambda}{16 k_{5}^{2} a_{-1}}, \\
a & =a,
\end{aligned}
$$

$$
\begin{aligned}
b & =\frac{\lambda-16 a a_{-1} k_{3} k_{5}}{16 k_{5} a_{-1}}, \\
\sigma & =\frac{16 k_{5}^{2} a_{-1}^{2}}{\lambda} .
\end{aligned}
$$

Set 3

$$
\begin{aligned}
a_{-1} & =a_{-1}, \\
a_{0} & =-\frac{k_{6}}{2 k_{5}}, \\
a_{1} & =0, \\
a & =a, \\
b & =\frac{\lambda-4 a a_{-1} k_{3} k_{5}}{4 k_{5} a_{-1}}, \\
\sigma & =\frac{4 k_{5}^{2} a_{-1}^{2}}{\lambda} .
\end{aligned}
$$

where $\lambda=4 k_{5} k_{7}-k_{6}^{2}$.

Case 2. When $k_{1} k_{2}<0$, let

$$
u(x, y, t)=u(\xi), \xi=a x+a \sqrt{-\frac{k_{1}}{k_{2}}} y+\frac{b t^{\alpha}}{\Gamma(1+\alpha)}, a \neq 0
$$

then Equation (21) is reduced to the ordinary differential equation as follows:

$$
\left(b+k_{3} a+a k_{4} \sqrt{-\frac{k_{1}}{k_{2}}}\right) u^{\prime}+k_{5} u^{2}+k_{6} u+k_{7}=0 .
$$

The solution of Equation (30) is in the form of (18). $n=1$ is taken from the homogeneous balance between the highest order derivative $u^{\prime}$ and the nonlinear term $u^{2}$. We obtain the solution of Equation (30) as Equation (24). Substituting Equation (24) together with its necessary derivatives into Equation (30), the algebraic equation is arranged according to the powers of the function $\varphi^{k}(\xi)$. Then, the following coefficients are obtained:

$$
\begin{array}{ll}
\varphi^{-2}: & -a \sqrt{-\frac{k_{1}}{k_{2}}} k_{4} \sigma a_{-1}-a k_{3} \sigma a_{-1}-b \sigma a_{-1}+k_{5} a_{-1}^{2}, \\
\varphi^{-1}: & 2 k_{5} a_{-1} a_{0}+a_{-1} k_{6} \\
\varphi^{0}: & a \sqrt{-\frac{k_{1}}{k_{2}} k_{4} \sigma a_{1}+a k_{3} \sigma a_{1}+b \sigma a_{1}-a \sqrt{-\frac{k_{1}}{k_{2}} k_{4} a_{-1}}} \\
\varphi^{1}: & 2 k_{5} a_{0} a_{1}+k_{6} a_{1},
\end{array}
$$




$$
\varphi^{2}: \quad a \sqrt{-\frac{k_{1}}{k_{2}}} k_{4} a_{1}+a k_{3} a_{1}+b a_{1}+k_{5} a_{1}^{2}
$$

Let the coefficients be zero. By solving the set of equations given above for $a_{-1}, a_{0}, a_{1}, a, b$, and $\sigma$, we obtain solution sets as follows:

Set 4

$$
\begin{aligned}
a_{-1} & =0, \\
a_{0} & =-\frac{k_{6}}{2 k_{5}}, \\
a_{1} & =a_{1}, \\
a & =a, \\
b & =-a k_{3}-a k_{4} \sqrt{-\frac{k_{1}}{k_{2}}}-a_{1} k_{5}, \\
\sigma & =\frac{\lambda}{4 a_{1}^{2} k_{5}^{2}} .
\end{aligned}
$$

Set 5

$$
\begin{gathered}
a_{-1}=a_{-1}, \\
a_{0}=-\frac{k_{6}}{2 k_{5}}, \\
a_{1}=\frac{-\lambda}{16 k_{5}^{2} a_{-1}}, \\
a=a, \\
b=\frac{\lambda-16 \mu}{16 k_{5} a_{-1}}, \\
\sigma=\frac{16 k_{5}^{2} a_{-1}^{2}}{\lambda} .
\end{gathered}
$$

Set 6

$$
\begin{aligned}
a_{-1} & =a_{-1}, \\
a_{0} & =-\frac{k_{6}}{2 k_{5}}, \\
a_{1} & =0, \\
a & =a, \\
b & =\frac{\lambda-4 \mu}{4 k_{5} a_{-1}}, \\
\sigma & =\frac{4 k_{5}^{2} a_{-1}^{2}}{\lambda} .
\end{aligned}
$$

where $\mu=a a_{-1} k_{3} k_{5}+a a_{-1} k_{4} k_{5} \sqrt{-k_{1} / k_{2}}$.

We find that $a_{-1}, a_{0}, a_{1}$, and $\sigma$ are equal in set 1 and set 4 , set 2 and set 5 , and set 3 and set 6 , respectively. In this study, the solutions of differential equations are symbolized as $u_{(i, j)(x, y, t)},\left(i, j \in Z^{+}\right)$, where $i$ denotes obtained set number and $j$ is the solution number of the Riccati equation, respectively. Thus, using set 1 to set 6 , we obtain the solution of Equation (21) as $u_{i, j}(x, y, t),(i=1,2, \cdots, 6 ; j=1,2, \cdots, 5)$. $u_{i, j}(x, y, t)$ is the following:

When $k_{1} k_{2}>0, \lambda=4 k_{5} k_{7}-k_{6}^{2}<0$, we have $\sigma<0$, then

$$
\begin{aligned}
& u_{1,1}(x, y, t)=-\frac{k_{6}}{2 k_{5}}-\frac{a_{1} \sqrt{-\lambda}}{2\left|a_{1} k_{5}\right|} \tanh \left[\frac{\sqrt{-\lambda}}{2\left|a_{1} k_{5}\right|}(a x\right. \\
& \left.\left.+i a \sqrt{\frac{k_{1}}{k_{2}}} y-\frac{\left(a k_{3}+a_{1} k_{5}\right) t^{\alpha}}{\Gamma(1+\alpha)}\right)\right] \text {, } \\
& u_{1,2}(x, y, t)=-\frac{k_{6}}{2 k_{5}}-\frac{a_{1} \sqrt{-\lambda}}{2\left|a_{1} k_{5}\right|} \operatorname{coth}\left[\frac{\sqrt{-\lambda}}{2\left|a_{1} k_{5}\right|}(a x\right.
\end{aligned}
$$

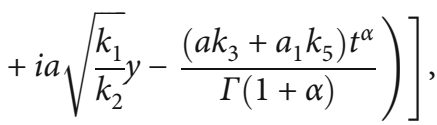

$$
\begin{aligned}
u_{2,1}(x, y, t)= & -\frac{a_{-1} \sqrt{-\lambda}}{4\left|k_{5} a_{-1}\right|} \operatorname{coth}\left[\frac { 4 | k _ { 5 } a _ { - 1 } | } { \sqrt { - \lambda } } \left(a x-i a \sqrt{\frac{k_{1}}{k_{2}} y}\right.\right. \\
& \left.\left.+\frac{\left(\lambda-16 a a_{-1} k_{3} k_{5}\right) t^{\alpha}}{16 k_{5} a_{-1} \Gamma(1+\alpha)}\right)\right]-\frac{k_{6}}{2 k_{5}} \\
& -\frac{\left|k_{5} a_{-1}\right| \sqrt{-\lambda}}{4 k_{5}^{2} a_{-1}} \tanh \left[\frac{4\left|k_{5} a_{-1}\right|}{\sqrt{-\lambda}}(a x\right. \\
& \left.\left.-i a \sqrt{\frac{k_{1}}{k_{2}}} y+\frac{\left(\lambda-16 a a_{-1} k_{3} k_{5}\right) t^{\alpha}}{16 k_{5} a_{-1} \Gamma(1+\alpha)}\right)\right],
\end{aligned}
$$$$
u_{2,2}(x, y, t)=-\frac{a_{-1} \sqrt{-\lambda}}{4\left|k_{5} a_{-1}\right|} \tanh \left[\frac { 4 | k _ { 5 } a _ { - 1 } | } { \sqrt { - \lambda } } \left(a x-i a \sqrt{\frac{k_{1}}{k_{2}} y}\right.\right.
$$$$
\left.\left.+\frac{\left(\lambda-16 a a_{-1} k_{3} k_{5}\right) t^{\alpha}}{16 k_{5} a_{-1} \Gamma(1+\alpha)}\right)\right]-\frac{k_{6}}{2 k_{5}}
$$$$
-\frac{\left|k_{5} a_{-1}\right| \sqrt{-\lambda}}{4 k_{5}^{2} a_{-1}} \operatorname{coth}\left[\frac{4\left|k_{5} a_{-1}\right|}{\sqrt{-\lambda}}(a x\right.
$$$$
\left.\left.-i a \sqrt{\frac{k_{1}}{k_{2}}} y+\frac{\left(\lambda-16 a a_{-1} k_{3} k_{5}\right) t^{\alpha}}{16 k_{5} a_{-1} \Gamma(1+\alpha)}\right)\right],
$$

$$
\begin{aligned}
u_{3,1}(x, y, t)= & \frac{a_{1} \sqrt{-\lambda}}{2\left|a_{1} k_{5} a_{-1}\right|} \operatorname{coth}\left[\frac{2\left|a_{1} k_{5} a_{-1}\right|}{\sqrt{-\lambda}}(a x\right. \\
& \left.\left.+i a \sqrt{\frac{k_{1}}{k_{2}} y}-\frac{\left(\lambda-4 a a_{-1} k_{3} k_{5}\right) t^{\alpha}}{4 k_{5} a_{-1} \Gamma(1+\alpha)}\right)\right]-\frac{k_{6}}{2 k_{5}},
\end{aligned}
$$




$$
\begin{aligned}
u_{3,2}(x, y, t)= & \frac{a_{-1} \sqrt{-\lambda}}{2\left|a_{1} k_{5} a_{-1}\right|} \tanh \left[\frac{2\left|a_{1} k_{5} a_{-1}\right|}{\sqrt{-\lambda}}(a x\right. \\
& \left.\left.+i a \sqrt{\frac{k_{1}}{k_{2}} y} y \frac{\left(\lambda-4 a a_{-1} k_{3} k_{5}\right) t^{\alpha}}{4 k_{5} a_{-1} \Gamma(1+\alpha)}\right)\right]-\frac{k_{6}}{2 k_{5}} .
\end{aligned}
$$

When $k_{1} k_{2}<0, \lambda=4 k_{5} k_{7}-k_{6}^{2}<0$, we have $\sigma<0$, then

$$
\begin{aligned}
u_{4,1}(x, y, t)= & -\frac{k_{6}}{2 k_{5}}-\frac{a_{1} \sqrt{-\lambda}}{2\left|a_{1} k_{5}\right|} \tanh \left[\frac { \sqrt { - \lambda } } { 2 | a _ { 1 } k _ { 5 } | } \left(a x+a \sqrt{-\frac{k_{1}}{k_{2}} y}\right.\right. \\
& \left.\left.-\frac{\left(a k_{3}+a k_{4} \sqrt{-k_{1} / k_{2}}+a_{1} k_{5}\right) t^{\alpha}}{\Gamma(1+\alpha)}\right)\right]
\end{aligned}
$$$$
u_{4,2}(x, y, t)=-\frac{k_{6}}{2 k_{5}}-\frac{a_{1} \sqrt{-\lambda}}{2\left|a_{1} k_{5}\right|} \operatorname{coth}\left[\frac { \sqrt { - \lambda } } { 2 | a _ { 1 } k _ { 5 } | } \left(a x+a \sqrt{-\frac{k_{1}}{k_{2}} y}\right.\right.
$$$$
\left.\left.-\frac{\left(a k_{3}+a k_{4} \sqrt{-k_{1} / k_{2}}+a_{1} k_{5}\right) t^{\alpha}}{\Gamma(1+\alpha)}\right)\right] \text {, }
$$$$
u_{5,1}(x, y, t)=-\frac{a_{-1} \sqrt{-\lambda}}{4\left|k_{5} a_{-1}\right|} \operatorname{coth}\left[\frac { 4 | k _ { 5 } a _ { - 1 } | } { \sqrt { - \lambda } } \left(a x-a \sqrt{-\frac{k_{1}}{k_{2}} y}\right.\right.
$$$$
\left.\left.+\frac{(\lambda-16 \mu) t^{\alpha}}{16 k_{5} a_{-1} \Gamma(1+\alpha)}\right)\right]-\frac{k_{6}}{2 k_{5}}
$$$$
-\frac{\left|k_{5} a_{-1}\right| \sqrt{-\lambda}}{4 k_{5}^{2} a_{-1}} \tanh \left[\frac{4\left|k_{5} a_{-1}\right|}{\sqrt{-\lambda}}(a x\right.
$$$$
\left.\left.-a \sqrt{-\frac{k_{1}}{k_{2}} y}+\frac{(\lambda-16 \mu) t^{\alpha}}{16 k_{5} a_{-1} \Gamma(1+\alpha)}\right)\right] \text {, }
$$$$
u_{5,2}(x, y, t)=-\frac{a_{-1} \sqrt{-\lambda}}{4\left|k_{5} a_{-1}\right|} \tanh \left[\frac { 4 | k _ { 5 } a _ { - 1 } | } { \sqrt { - \lambda } } \left(a x-a \sqrt{-\frac{k_{1}}{k_{2}} y}\right.\right.
$$$$
\left.\left.+\frac{(\lambda-16 \mu) t^{\alpha}}{16 k_{5} a_{-1} \Gamma(1+\alpha)}\right)\right]-\frac{k_{6}}{2 k_{5}}
$$$$
-\frac{\left|k_{5} a_{-1}\right| \sqrt{-\lambda}}{4 k_{5}^{2} a_{-1}} \operatorname{coth}\left[\frac{4\left|k_{5} a_{-1}\right|}{\sqrt{-\lambda}}(a x\right.
$$$$
\left.\left.-a \sqrt{-\frac{k_{1}}{k_{2}} y}+\frac{(\lambda-16 \mu) t^{\alpha}}{16 k_{5} a_{-1} \Gamma(1+\alpha)}\right)\right] \text {, }
$$

$$
\begin{aligned}
u_{6,1}(x, y, t)= & \frac{a_{-1} \sqrt{-\lambda}}{2\left|a_{1} k_{5} a_{-1}\right|} \operatorname{coth}\left[\frac { 2 | a _ { 1 } k _ { 5 } a _ { - 1 } | } { \sqrt { - \lambda } } \left(a x+a \sqrt{-\frac{k_{1}}{k_{2}} y}\right.\right. \\
& \left.\left.+\frac{(\lambda-4 \mu) t^{\alpha}}{4 k_{5} a_{-1} \Gamma(1+\alpha)}\right)\right]-\frac{k_{6}}{2 k_{5}}
\end{aligned}
$$

$$
\begin{aligned}
u_{6,2}(x, y, t)= & \frac{a_{-1} \sqrt{-\lambda}}{2\left|a_{1} k_{5} a_{-1}\right|} \tanh \left[\frac { 2 | a _ { 1 } k _ { 5 } a _ { - 1 } | } { \sqrt { - \lambda } } \left(a x+a \sqrt{-\frac{k_{1}}{k_{2}} y}\right.\right. \\
& \left.\left.+\frac{(\lambda-4 \mu) t^{\alpha}}{4 k_{5} a_{-1} \Gamma(1+\alpha)}\right)\right]-\frac{k_{6}}{2 k_{5}} .
\end{aligned}
$$

When $k_{1} k_{2}<0$ and $\lambda=4 k_{5} k_{7}-k_{6}^{2}<0$, we have $\sigma<0$, then

$$
\begin{aligned}
& u_{1,3}(x, y, t)=-\frac{k_{6}}{2 k_{5}}+\frac{a_{1} \sqrt{\lambda}}{2\left|a_{1} k_{5}\right|} \tan \left[\frac{\sqrt{\lambda}}{2\left|a_{1} k_{5}\right|}(a x\right.
\end{aligned}
$$

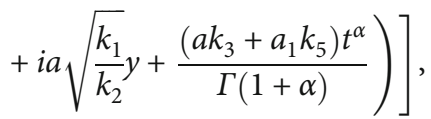

$$
\begin{aligned}
& u_{1,4}(x, y, t)=-\frac{k_{6}}{2 k_{5}}+\frac{a_{1} \sqrt{\lambda}}{2\left|a_{1} k_{5}\right|} \cot \left[\frac{\sqrt{\lambda}}{2\left|a_{1} k_{5}\right|}(a x\right.
\end{aligned}
$$

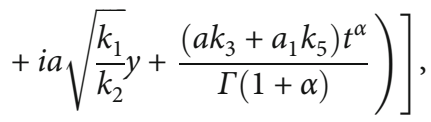

$$
\begin{aligned}
& u_{2,3}(x, y, t)=-\frac{a_{-1} \sqrt{\lambda}}{4\left|k_{5} a_{-1}\right|} \cot \left[\frac { 4 | k _ { 5 } a _ { - 1 } | } { \sqrt { \lambda } } \left(a x-i a \sqrt{\frac{k_{1}}{k_{2}} y}\right.\right. \\
& \left.\left.+\frac{\left(\lambda-16 a a_{-1} k_{3} k_{5}\right) t^{\alpha}}{16 k_{5} a_{-1} \Gamma(1+\alpha)}\right)\right]-\frac{k_{6}}{2 k_{5}} \\
& +\frac{\left|k_{5} a_{-1}\right| \sqrt{\lambda}}{4 k_{5}^{2} a_{-1}} \tan \left[\frac{4\left|k_{5} a_{-1}\right|}{\sqrt{\lambda}}(a x\right.
\end{aligned}
$$

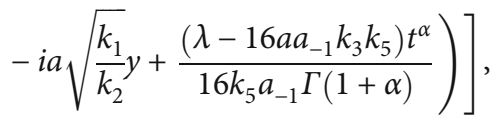

$$
\begin{aligned}
& u_{2,4}(x, y, t)=-\frac{a_{-1} \sqrt{\lambda}}{4\left|k_{5} a_{-1}\right|} \tan \left[\frac { 4 | k _ { 5 } a _ { - 1 } | } { \sqrt { \lambda } } \left(a x-i a \sqrt{\frac{k_{1}}{k_{2}} y}\right.\right. \\
& \left.\left.+\frac{\left(\lambda-16 a a_{-1} k_{3} k_{5}\right) t^{\alpha}}{16 k_{5} a_{-1} \Gamma(1+\alpha)}\right)\right]-\frac{k_{6}}{2 k_{5}} \\
& -\frac{\left|k_{5} a_{-1}\right| \sqrt{\lambda}}{4 k_{5}^{2} a_{-1}} \cot \left[\frac{4\left|k_{5} a_{-1}\right|}{\sqrt{\lambda}}(a x\right.
\end{aligned}
$$

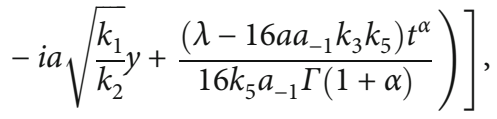

$$
\begin{aligned}
& u_{3,3}(x, y, t)=-\frac{a_{-1} \sqrt{\lambda}}{2\left|a_{1} k_{5} a_{-1}\right|} \cot \left[\frac{2\left|a_{1} k_{5} a_{-1}\right|}{\sqrt{\lambda}}(a x\right.
\end{aligned}
$$

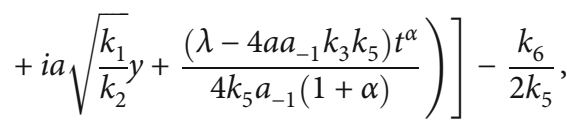

$u_{3,4}(x, y, t)=-\left(a_{-1} \sqrt{\lambda} / 2\left|a_{1} k_{5} a_{-1}\right|\right) \tan \left[\left(2\left|a_{1} k_{5} a_{-1}\right| / \sqrt{\lambda}\right)\right.$ $\left.\left(a x+i a \sqrt{k_{1} / k_{2}} y+\left(\left(\lambda-4 a a_{-1} k_{3} k_{5}\right) t^{\alpha} / 4 k_{5} a_{-1}(1+\alpha)\right)\right)\right]-k_{6} /$ $2 k_{5}$, 
When $k_{1} k_{2}<0$ and $\lambda=4 k_{5} k_{7}-k_{6}^{2}<0$, we have $\sigma<0$, then

$$
\begin{aligned}
u_{4,3}(x, y, t)= & -\frac{k_{6}}{2 k_{5}}+\frac{a_{1} \sqrt{\lambda}}{2\left|a_{1} k_{5}\right|} \tan \left[\frac { \sqrt { \lambda } } { 4 | a _ { 1 } k _ { 5 } | } \left(a x+a \sqrt{-\frac{k_{1}}{k_{2}} y}\right.\right. \\
& \left.\left.-\frac{\left(a k_{3}+a k_{4} \sqrt{-k_{1} / k_{2}}+a_{1} k_{5}\right) t^{\alpha}}{\Gamma(1+\alpha)}\right)\right], \\
u_{4,4}(x, y, t)= & -\frac{k_{6}}{2 k_{5}}-\frac{a_{1} \sqrt{\lambda}}{2\left|a_{1} k_{5}\right|} \cot \left[\frac { \sqrt { \lambda } } { 2 | a _ { 1 } k _ { 5 } | } \left(a x+a \sqrt{-\frac{k_{1}}{k_{2}} y}\right.\right. \\
& \left.\left.-\frac{\left(a k_{3}+a k_{4} \sqrt{-k_{1} / k_{2}}+a_{1} k_{5}\right) t^{\alpha}}{\Gamma(1+\alpha)}\right)\right] \\
u_{5,3}(x, y, t)= & -\frac{a_{-1} \sqrt{\lambda}}{4\left|k_{5} a_{-1}\right|} \cot \left[\frac { 4 | k _ { 5 } a _ { - 1 } | } { \sqrt { \lambda } } \left(a x+a \sqrt{-\frac{k_{1}}{k_{2}} y}\right.\right. \\
& \left.\left.+\frac{(\lambda-16 \mu) t^{\alpha}}{16 k_{5} a_{-1} \Gamma(1+\alpha)}\right)\right]-\frac{k_{6}}{2 k_{5}} \\
& +\frac{\left|k_{5} a_{-1}\right| \sqrt{\lambda}}{4 k_{5}^{2} a_{-1}} \tan \left[\frac{4\left|k_{5} a_{-1}\right|}{\sqrt{\lambda}}\right. \\
& \cdot\left(a x+a \sqrt{\left.\left.-\frac{k_{1}}{k_{2}} y+\frac{(\lambda-16 \mu) t^{\alpha}}{16 k_{5} a_{-1} \Gamma(1+\alpha)}\right)\right]}\right.
\end{aligned}
$$

$$
u_{5,4}(x, y, t)=-\frac{a_{-1} \sqrt{\lambda}}{4\left|k_{5} a_{-1}\right|} \tan \left[\frac { 4 | k _ { 5 } a _ { - 1 } | } { \sqrt { \lambda } } \left(a x+a \sqrt{-\frac{k_{1}}{k_{2}}} y\right.\right.
$$$$
\left.\left.+\frac{(\lambda-16 \mu) t^{\alpha}}{16 k_{5} a_{-1} \Gamma(1+\alpha)}\right)\right]-\frac{k_{6}}{2 k_{5}}
$$$$
-\frac{\left|k_{5} a_{-1}\right| \sqrt{\lambda}}{4 k_{5}^{2} a_{-1}} \cot \left[\frac{4\left|k_{5} a_{-1}\right|}{\sqrt{\lambda}}\right.
$$$$
\left.\cdot\left(a x+a \sqrt{-\frac{k_{1}}{k_{2}}} y+\frac{(\lambda-16 \mu) t^{\alpha}}{16 k_{5} a_{-1} \Gamma(1+\alpha)}\right)\right],
$$$$
u_{6,3}(x, y, t)=\frac{a_{-1} \sqrt{\lambda}}{2\left|k_{5} a_{-1}\right|} \cot \left[\frac { 2 | k _ { 5 } a _ { - 1 } | } { \sqrt { \lambda } } \left(a x+a \sqrt{-\frac{k_{1}}{k_{2}}} y\right.\right.
$$$$
\left.\left.+\frac{(\lambda-4 \mu) t^{\alpha}}{4 k_{5} a_{-1} \Gamma(1+\alpha)}\right)\right]-\frac{k_{6}}{2 k_{5}},
$$$$
u_{6,4}(x, y, t)=\frac{a_{-1} \sqrt{\lambda}}{2\left|k_{5} a_{-1}\right|} \tan \left[\frac { 2 | k _ { 5 } a _ { - 1 } | } { \sqrt { \lambda } } \left(a x+a \sqrt{-\frac{k_{1}}{k_{2}} y}\right.\right.
$$$$
\left.\left.+\frac{(\lambda-4 \mu) t^{\alpha}}{4 k_{5} a_{-1} \Gamma(1+\alpha)}\right)\right]-\frac{k_{6}}{2 k_{5}} \text {. }
$$

When $\lambda=4 k_{5} k_{7}-k_{6}^{2}=0$, we have $\sigma=0$, then

$$
\begin{aligned}
& u_{1,5}(x, y, t)=-\frac{k_{6}}{2 k_{5}}-\frac{\Gamma(1+\alpha) a_{1}}{\left[a x+i a \sqrt{\left(k_{1} / k_{2}\right)} y-\left(\left(a k_{3}+a_{1} k_{5}\right) t^{\alpha} / \Gamma(1+\alpha)\right)\right]^{\alpha}+\omega}, \\
& u_{2,5}(x, y, t)=-\frac{a_{-1}\left[a x+i a \sqrt{\left(k_{1} / k_{2}\right)} y-\left(a k_{3} t^{\alpha} / \Gamma(1+\alpha)\right)\right]^{\alpha}+a_{-1} \omega}{\Gamma(1+\alpha)}-\frac{k_{6}}{2 k_{5}}=u_{3,5}(x, y, t), \\
& u_{4,5}(x, y, t)=-\frac{k_{6}}{2 k_{5}\left[a x+a \sqrt{\left(-k_{1} / k_{2}\right)} y-\left(\left(a k_{3}+a k_{3} \sqrt{-k_{1} / k_{2}}+a_{1} k_{5}\right) t^{\alpha} / \Gamma(1+\alpha)\right)\right]^{\alpha}+\omega}, \\
& u_{5,5}(x, y, t)=\frac{-a_{-1}}{\Gamma(1+\alpha)}\left[a^{\alpha}\left(x+\sqrt{\left(-k_{1} / k_{2}\right)} y-\frac{\left(k_{3}+k_{4} \sqrt{-k_{1} / k_{2}}\right) t^{\alpha}}{\Gamma(1+\alpha)}\right)^{\alpha}+\omega\right]-\frac{k_{6}}{2 k_{5}} .
\end{aligned}
$$

Solutions $u_{i, 1}, u_{i, 2}(i=1,2, \cdots, 6)$ describe the soliton. Solitons exist everywhere in the nature; they are special kinds of solitary waves. $u_{2,1}, u_{2,2}, u_{5,1}$ and $u_{5,2}$ describe the multiple soliton solutions. Solutions $u_{i, 3}$ and $u_{i, 4}(i=1,2, \cdots, 6)$ represent the exact periodic traveling wave solutions. Periodic solutions are traveling wave solutions.

Figures 1-6 present the solutions: $u_{1,1}, u_{2,1}, u_{1,3}, u_{2,3}$, $u_{1,5}$, and $u_{5,5}$ of the generalized time fractional biological population model with $-10<x<10,0<t<10$. Solution $u_{1,1}$ is presented for values $\alpha=0.5, k_{1}=k_{2}=1, k_{3}=k_{4}=0$, $k_{5}=2, k_{6}=3, k_{7}=1, a_{1}=1, a=2$, and $y=0$; solution $u_{2,1}$ is presented for values $\alpha=0.5, k_{1}=k_{2}=1, k_{3}=k_{4}=0, k_{5}=2$, $k_{6}=3, k_{7}=1, a_{-1}=1, a=2$, and $y=0$; solution $u_{1,3}$ is presented for values $\alpha=0.5, k_{1}=k_{2}=1, k_{3}=k_{4}=0, k_{5}=2, k_{6}$ $=2, k_{7}=1, a_{1}=1, a=2$, and $y=0$; solution $u_{2,3}$ is presented for values $\alpha=0.5, k_{1}=k_{2}=1, k_{3}=\mathrm{k}_{4}=0, k_{5}=2, k_{6}=3, k_{7}=$ 


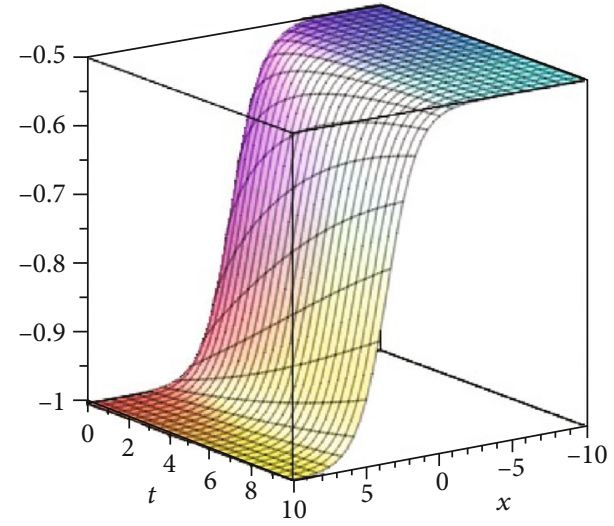

Figure 1: $u_{1,1}(x, 0, t)$ of Equation (21).

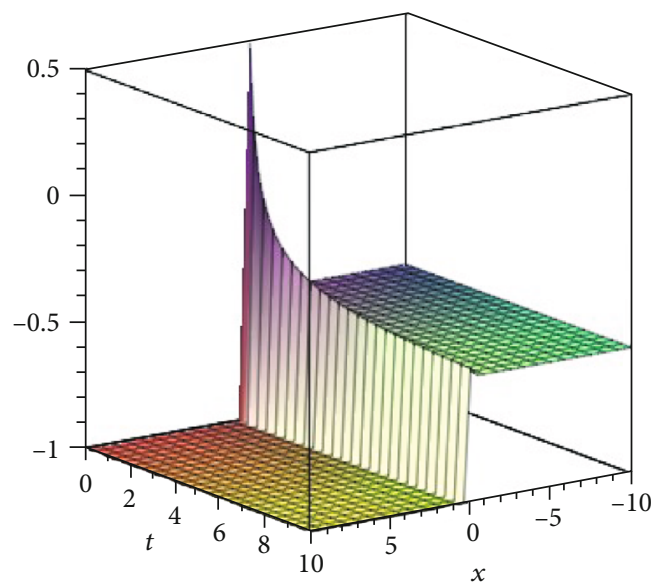

FIgURE 2: $u_{2,1}(x, 0, t)$ of Equation (21).

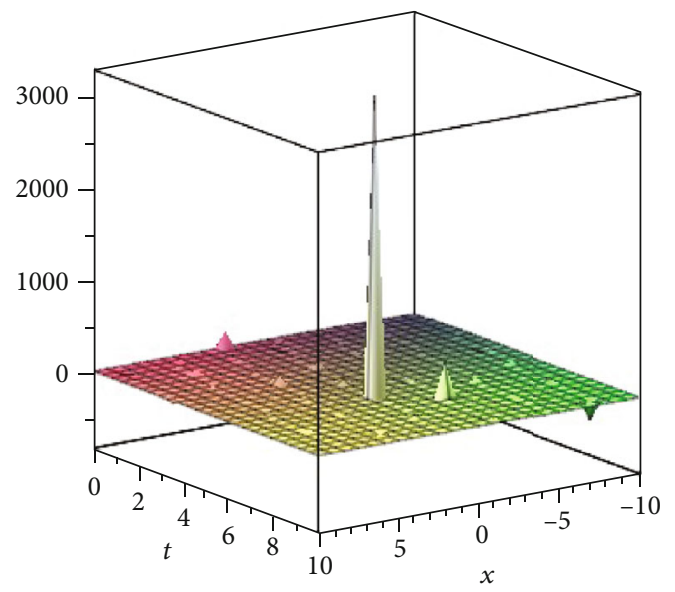

Figure 3: $u_{1,3}(x, 0, t)$ of Equation (21).

$1, a_{-1}=1, a=2$, and $y=0$; solution $u_{1,5}$ is presented for values $\alpha=0.5, k_{1}=k_{2}=1, k_{3}=k_{4}=0, k_{5}=2, k_{6}=\sqrt{8}, k_{7}=1, a=2$, $a_{1}=1, y=0$, and $\omega=0$; solution $u_{5,5}$ is presented for values $\alpha=0.5, k_{1}=1, k_{2}=-1, k_{3}=0, k_{4}=1, k_{5}=2, k_{6}=\sqrt{8}, k_{7}=1$, $a_{1}=1, a=2, y=0$, and $\omega=0$.

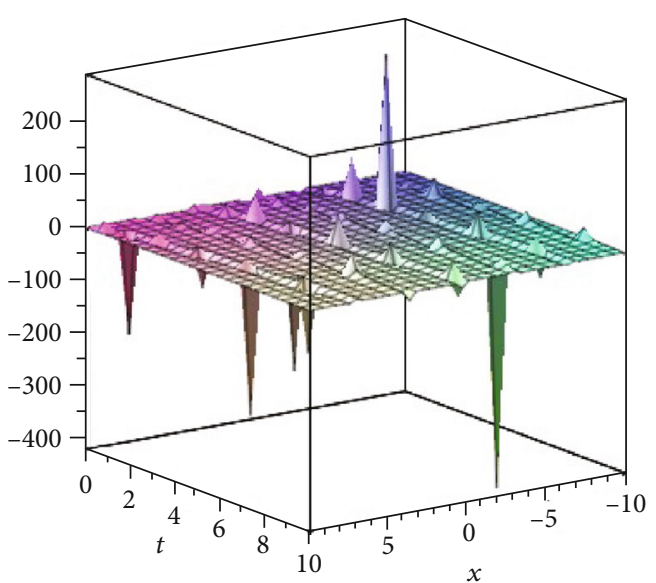

Figure 4: $u_{2,3}(x, 0, t)$ of Equation (21).

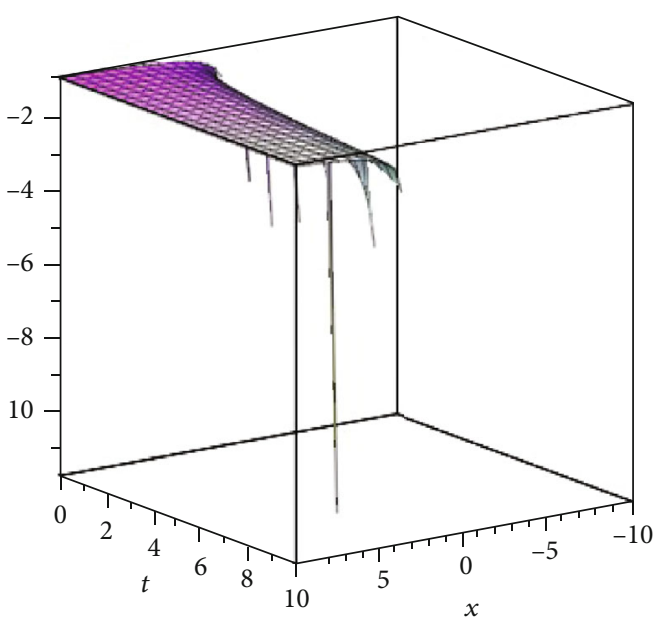

Figure 5: $u_{1,5}(x, 0, t)$ of Equation (21).

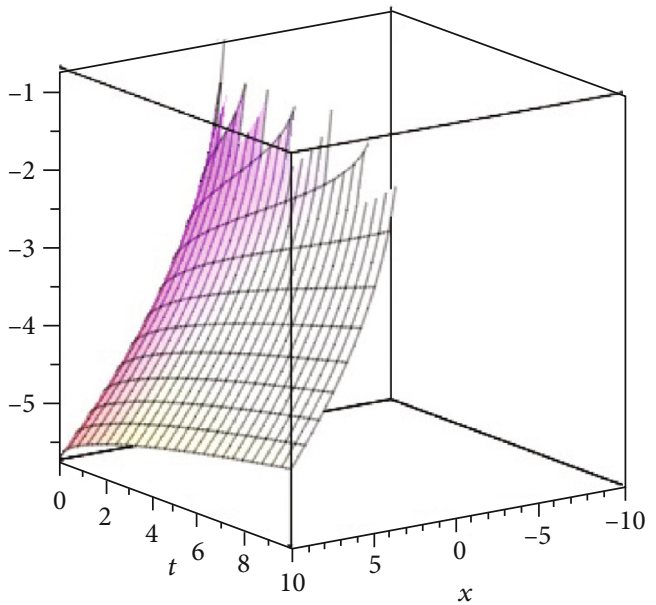

Figure 6: $u_{5,5}(x, 0, t)$ of Equation (21).

When $k_{1}=-1, k_{2}=-1, k_{3}=k_{4}=k_{6}=0, k_{5}=-h \neq 0$, and $k_{7}=r h \neq 0$, Equation (21) is the time fractional biological population model [39]

$$
D_{t}^{\alpha} u=\left(u^{2}\right)_{x x}+\left(u^{2}\right)_{y y}+h\left(u^{2}-r\right) .
$$


We denote

$$
u(x, y, t)=u(\xi), \xi=a x+i a y+\frac{b t^{\alpha}}{\Gamma(1+\alpha)}, a \neq 0
$$

and find the solution of Equation (40) in the form of $u$ $(\xi)=a_{-1} \varphi^{-1}+a_{0}+a_{1} \varphi$. Then, by solving the set of equations given above for $a_{-1}, a_{0}, a_{1}, a, b$, and $\sigma$, we obtain solution sets as follows:

Set 1

$$
\begin{aligned}
a_{-1} & =0, \\
a_{0} & =0, \\
a_{1} & \neq 0, \\
\sigma & =-\frac{r}{a_{1}^{2}} .
\end{aligned}
$$

Let $a_{1}=\mathrm{c} / h$, then $\sigma=-r h^{2} / c^{2}$, we can obtain set 1 in [42]. Set 2

$$
\begin{aligned}
a_{-1} & \neq 0, \\
a_{0} & =0, \\
a_{1} & =\frac{r}{4 a_{-1}}, \\
\sigma & =-\frac{4 a_{-1}^{2}}{r} .
\end{aligned}
$$
[42].

Let $a_{-1}=r h / 4 c$, then $\sigma=-r h^{2} / 4 c^{2}$, we can obtain set 3 in

Set 3

$$
\begin{aligned}
a_{-1} & \neq 0, \\
a_{0} & =0, \\
a_{1} & =0, \\
\sigma & =-\frac{a_{-1}^{2}}{r} .
\end{aligned}
$$
[42].

Let $a_{-1}=r h / c$, then $\sigma=-r h^{2} / c^{2}$, we can obtain set 2 in

Clearly, we get more solutions of the time fractional biological population model (40) than the literature [42].

3.2. The Generalized Compound KdV-Burgers Equation. The generalized compound $\mathrm{KdV}$-Burgers equation is given by

$$
D_{t}^{\alpha} u+k_{1} u u_{x}+k_{2} u^{2} u_{x}+k_{3} u_{x x}+k_{4} u_{x x x}=0 \text {, }
$$

Let

$$
u(x, t)=u(\xi), \xi=a x+\frac{b t^{\alpha}}{\Gamma(1+\alpha)}, a \neq 0
$$

then Equation (45) is reduced to ordinary differential equation as

$$
b u^{\prime}+k_{1} a u u^{\prime}+k_{2} a u^{2} u^{\prime}+k_{3} a^{2} u^{\prime \prime}+k_{4} a^{3} u^{\prime \prime \prime}=0 .
$$

The solution of Equation (45) is in the form of (18), and here, $n=1$ is taken from the homogeneous balance between the highest order derivative $u^{\prime \prime \prime}$ and the nonlinear term $u^{2} u^{\prime}$. We obtain the solution of Equation (45) as follows:

$$
u(\xi)=a_{-1} \varphi^{-1}+a_{0}+a_{1} \varphi
$$

Substituting Equation (48) together with its necessary derivatives into Equation (47), the algebraic equation is arranged according to the powers of the function $\varphi^{k}(\xi)$. Then, the following coefficients are obtained:

$$
\begin{aligned}
\varphi^{-4}: & -6 a^{3} k_{4} \sigma^{3} a_{-1}-a k_{2} \sigma a_{-1}^{3}, \\
\varphi^{-3}: & 2 a^{2} k_{3} \sigma^{2} a_{-1}-2 a k_{2} \sigma a_{-1}^{2} a_{0}-a k_{1} \sigma a_{-1}^{2}, \\
\varphi^{-2}: & b \sigma a_{-1}-a k_{1} \sigma a_{-1} a_{0}-a k_{2} a_{-1}^{3}-a k_{2} \sigma a_{-1} a_{0}^{2} \\
& -a k_{2} \sigma a_{-1}^{2} a_{1}-8 a^{3} k_{4} \sigma^{2} a_{-1}, \\
\varphi^{-1}:- & a k_{1} a_{-1}^{2}-2 a k_{2} a_{-1}^{2} a_{0}+2 a^{2} k_{3} \sigma a_{-1}, \\
\varphi^{0}: & b a_{-1}-b \sigma a_{1}+a k_{1} \sigma a_{0} a_{1}-a k_{1} a_{-1} a_{0} \\
& +a k_{2} \sigma a_{0}^{2} a_{1}+a k_{2} \sigma a_{-1} a_{1}^{2}-a k_{2} a_{0}^{2} a_{-1} \\
& -a k_{2} a_{-1}^{2} a_{1}+2 a^{3} k_{4} \sigma^{2} a_{1}-2 a^{3} k_{4} \sigma a_{-1}, \\
\varphi^{1}: & a k_{1} \sigma a_{1}^{2}+2 a k_{2} \sigma a_{0} a_{1}^{2}+2 a^{2} k_{3} \sigma a_{1}, \\
\varphi^{2}: & -b a_{1}+a k_{1} a_{0} a_{1}+a k_{2} a_{0}^{2} a_{1}+a k_{2} a_{-1} a_{1}^{2} \\
& +a k_{2} \sigma a_{1}^{3}+8 a^{3} k_{4} \sigma a_{1}, \\
\varphi^{3}: & 2 a k_{2} a_{0} a_{1}^{2}+2 a^{2} k_{3} a_{1}+a k_{1} a_{1}^{2}, \\
\varphi^{4}: & 6 a^{3} k_{4} a_{1}+a k_{2} a_{1}^{3} .
\end{aligned}
$$

Let the coefficients of $\varphi^{k}(\xi)$ to be zero. By solving the set of equations given above for $a_{-1}, a_{0}, a_{1}, a, b$, and $\sigma$, we obtain solution sets as follows:

When $k_{2} k_{4}<0$, it denotes $\mu=3 a k_{1}^{2} k_{4}+2 a k_{2} k_{3}^{2}-12 b k_{2}$ $k_{4}$ and $\lambda_{1}= \pm \sqrt{-6 k_{4} / k_{2}}, \lambda_{2}=-k_{1} / 2 \pm\left(\left(\left|k_{3}\right| \sqrt{-6 k_{2} k_{4}}\right) / 6 k_{4}\right)$, we have

Set 1

$$
\begin{aligned}
a_{-1} & =-\frac{\lambda_{1} \mu}{96 a^{2} k_{2} k_{4}^{2}}, \\
a_{0} & =-\frac{\lambda_{1} k_{1}+2 k_{3}}{2 \lambda_{1} k_{2}}, \\
a_{1} & =a \lambda_{1}, \\
a & =a, \\
b & =b, \\
\sigma & =\frac{\mu}{96 a^{3} k_{2} k_{4}^{2}} .
\end{aligned}
$$


Set 2

$$
\begin{aligned}
a_{-1} & =0, \\
a_{0} & =-\frac{\lambda_{1} k_{1}+2 k_{3}}{2 \lambda_{1} k_{2}}, \\
a_{1} & =a \lambda_{1}, \\
a & =a, \\
b & =b, \\
\sigma & =\frac{\mu}{24 a^{3} k_{2} k_{4}^{2}} .
\end{aligned}
$$

Set 3

$$
\begin{aligned}
a_{-1} & =-\frac{\mu k_{3}}{12 a^{2} k_{2} k_{4}^{2}\left(2 \lambda_{2}+k_{1}\right)}, \\
a_{0} & =\frac{\lambda_{2}}{k_{2}}, \\
a_{1} & =0, \\
a & =a, \\
b & =b, \\
\sigma & =\frac{\mu}{24 a^{3} k_{2} k_{4}^{2}} .
\end{aligned}
$$

Thus, we obtain the solution of Equation (45) as $u_{i, j}$ $(x, t),(i=1,2,3 ; j=1,2,3,4,5)$. Using set 1 to set $3, u_{i, j}$ $(x, t)$ is as follows:

When $k_{2} k_{4}<0$ and $a \mu k_{2}<0$, we have $\sigma<0$, then

$$
\begin{aligned}
u_{1,1}(x, t)= & \frac{\lambda_{1} \mu|a|}{24 a^{2} k_{4}} \sqrt{\frac{-6 a}{\mu k_{2}}} \operatorname{coth}\left[\frac{1}{4\left|a k_{4}\right|} \sqrt{\frac{-\mu}{6 a k_{2}}}\right. \\
& \left.\cdot\left(a x+\frac{b t^{\alpha}}{\Gamma(1+\alpha)}\right)\right]-\frac{\lambda_{1} k_{1}+2 k_{3}}{2 \lambda_{1} k_{2}} \\
& -\frac{a \lambda_{1}}{4\left|a k_{4}\right|} \sqrt{\frac{-\mu}{6 a k_{2}}} \tanh \left[\frac{1}{4\left|a k_{4}\right|}\right. \\
& \left.\cdot \sqrt{\frac{-\mu}{6 a k_{2}}}\left(a x+\frac{b t^{\alpha}}{\Gamma(1+\alpha)}\right)\right] \\
u_{1,2}(x, t)= & \frac{\lambda_{1} \mu|a|}{24 a^{2} k_{4}} \sqrt{\frac{-6 a}{\mu k_{2}}} \tanh \left[\frac{1}{4\left|a k_{4}\right|} \sqrt{\frac{-\mu}{6 a k_{2}}}\right. \\
& \left.\cdot\left(a x+\frac{b t^{\alpha}}{\Gamma(1+\alpha)}\right)\right]-\frac{\lambda_{1} k_{1}+2 k_{3}}{2 \lambda_{1} k_{2}} \\
& -\frac{a \lambda_{1}}{4\left|a k_{4}\right|} \sqrt{\frac{-\mu}{6 a k_{2}}} \operatorname{coth}\left[\frac{1}{4\left|a k_{4}\right|}\right. \\
& \left.\cdot \sqrt{\frac{-\mu}{6 a k_{2}}}\left(a x+\frac{b t^{\alpha}}{\Gamma(1+\alpha)}\right)\right]
\end{aligned}
$$

$$
\begin{aligned}
u_{2,1}(\mathrm{x}, t)= & -\frac{\lambda_{1} k_{1}+2 k_{3}}{2 \lambda_{1} k_{2}}-\frac{a \lambda_{1}}{2\left|a k_{4}\right|} \sqrt{\frac{-\mu}{6 a k_{2}}} \tanh \\
& \cdot\left[\frac{1}{2\left|a k_{4}\right|} \sqrt{\frac{-\mu}{6 a k_{2}}}\left(a x+\frac{b t^{\alpha}}{\Gamma(1+\alpha)}\right)\right] \\
u_{2,2}(x, t)= & -\frac{\lambda_{1} k_{1}+2 k_{3}}{2 \lambda_{1} k_{2}}-\frac{a \lambda_{1}}{2\left|a k_{4}\right|} \sqrt{\frac{-\mu}{6 a k_{2}}} \operatorname{coth}, \\
u_{3,1}(x, t)= & \frac{\mu k_{3}|a|}{6 a^{2} k_{4}\left(2 \lambda_{2}+k_{1}\right)} \sqrt{\frac{-6 a}{\mu k_{2}}} \operatorname{coth}+\frac{\lambda_{2}}{k_{2}}, \\
u_{3,2}(x, t)= & \frac{\mu k_{3}|a|}{6 a^{2} k_{4}\left(2 \lambda_{2}+k_{1}\right)} \sqrt{\frac{-6 a}{\mu k_{2}}} \tanh +\frac{\lambda_{2}}{k_{2}} .
\end{aligned}
$$

When $k_{2} k_{4}<0$ and $a \mu k_{2}>0$, we have $\sigma>0$, then

$$
\begin{aligned}
u_{1,3}(x, t)= & -\frac{\lambda_{1} \mu|a|}{24 a^{2} k_{4}} \sqrt{\frac{6 a}{\mu k_{2}}} \cot \left[\frac{1}{4\left|a k_{4}\right|} \sqrt{\frac{\mu}{6 a k_{2}}}\right. \\
& \left.\cdot\left(a x+\frac{b t^{\alpha}}{\Gamma(1+\alpha)}\right)\right]-\frac{\lambda_{1} k_{1}+2 k_{3}}{2 \lambda_{1} k_{2}} \\
& +\frac{a \lambda_{1}}{4\left|a k_{4}\right|} \sqrt{\frac{\mu}{6 a k_{2}}} \tan \left[\frac{1}{4\left|a k_{4}\right|} \sqrt{\frac{\mu}{6 a k_{2}}}\right. \\
& \left.\cdot\left(a x+\frac{b t^{\alpha}}{\Gamma(1+\alpha)}\right)\right]
\end{aligned}
$$

$$
\begin{aligned}
u_{1,4}(x, t)= & \frac{\lambda_{1} \mu|a|}{24 a^{2} k_{4}} \sqrt{\frac{6 a}{\mu k_{2}}} \tan \left[\frac{1}{4\left|a k_{4}\right|} \sqrt{\frac{\mu}{6 a k_{2}}}\right. \\
& \left.\cdot\left(a x+\frac{b t^{\alpha}}{\Gamma(1+\alpha)}\right)\right]-\frac{\lambda_{1} k_{1}+2 k_{3}}{2 \lambda_{1} k_{2}} \\
& -\frac{a \lambda_{1}}{4\left|a k_{4}\right|} \sqrt{\frac{\mu}{6 a k_{2}}} \cot \left[\frac{1}{4\left|a k_{4}\right|} \sqrt{\frac{\mu}{6 a k_{2}}}\right. \\
& \left.\cdot\left(a x+\frac{b t^{\alpha}}{\Gamma(1+\alpha)}\right)\right], \\
u_{2,3}(x, t)= & -\frac{\lambda_{1} k_{1}+2 k_{3}}{2 \lambda_{1} k_{2}}+\frac{a \lambda_{1}}{2\left|a k_{4}\right|} \sqrt{\frac{\mu}{6 a k_{2}}} \tan \\
& \cdot\left[\frac{1}{2\left|a k_{4}\right|} \sqrt{\frac{\mu}{6 a k_{2}}}\left(a x+\frac{b t^{\alpha}}{\Gamma(1+\alpha)}\right)\right], \\
u_{2,4}(x, t)= & -\frac{\lambda_{1} k_{1}+2 k_{3}}{2 \lambda_{1} k_{2}}-\frac{a \lambda_{1}}{2\left|a k_{4}\right|} \sqrt{\frac{\mu}{6 a k_{2}}} \operatorname{coth} \\
& \cdot\left[\frac{1}{2\left|a k_{4}\right|} \sqrt{\frac{\mu}{6 a k_{2}}}\left(a x+\frac{b t^{\alpha}}{\Gamma(1+\alpha)}\right)\right], \\
u_{3,3}(x, t)= & -\frac{\mu k_{3}}{6 a^{2} k_{4}\left(2 \lambda_{2}+k_{1}\right)} \sqrt{\frac{6 a^{3}}{\mu k_{2}}} \cot \\
& \cdot\left[\frac{1}{2\left|k_{4}\right|} \sqrt{\frac{\mu}{6 a^{3} k_{2}}}\left(a x+\frac{b t^{\alpha}}{\Gamma(1+\alpha)}\right)\right]+\frac{\lambda_{2}}{k_{2}},
\end{aligned}
$$




$$
\begin{aligned}
u_{3,4}(x, t)= & \frac{\mu k_{3}|a|}{6 a^{2} k_{4}\left(2 \lambda_{2}+k_{1}\right)} \sqrt{\frac{6 a}{\mu k_{2}}} \tan \\
& \cdot\left[\frac{1}{2\left|a k_{4}\right|} \sqrt{\frac{\mu}{6 a k_{2}}}\left(a x+\frac{b t^{\alpha}}{\Gamma(1+\alpha)}\right)\right]+\frac{\lambda_{2}}{k_{2}} .
\end{aligned}
$$

When $k_{2} k_{4}<0$ and $\mu=0$, we have $\sigma=0$, then

$$
\begin{aligned}
u_{1,5}(x, t) & =-\frac{\lambda_{1} k_{1}+2 k_{3}}{2 \lambda_{1} k_{2}}+\frac{a \lambda_{1} \Gamma(1+\alpha)}{\left(a x+\left(b t^{\alpha} / \Gamma(1+\alpha)\right)\right)^{\alpha}+\omega} \\
& =u_{2,5}(x, t) .
\end{aligned}
$$

Solutions $u_{i, 1}$ and $u_{i, 2}(i=1,2,3)$ described the soliton. $u_{1,1}$ and $u_{1,2}$ describe the multiple soliton solutions. Solutions $u_{i, 3}$ and $u_{i, 4}(i=1,2,3)$ represent the exact periodic traveling wave solutions.

Figures $7-10$ present the solutions: $u_{1,1}, u_{2,1}, u_{1,3}$, and $u_{2,3}$ of the generalized compound $\mathrm{KdV}$-Burgers equation with $10<x<10,0<t<1$. Solutions $u_{1,1}$ and $u_{2,1}$ are presented for values $\alpha=0.5, k_{1}=k_{2}=1, k_{3}=k_{4}=-1, a=-4, b=1$, and $y=0$; solutions $u_{1,3}$ and $u_{2,3}$ are presented for values $\alpha=0.5$, $k_{1}=k_{2}=1, k_{3}=k_{4}=-1, a=4, \mathrm{~b}=1$, and $y=0$.

3.3. The Space-Time Fractional Regularized Long-Wave Equation. Consider the space-time fractional regularized long-wave equation as follows:

$$
D_{t}^{\alpha} u+k_{1} D_{x}^{\alpha} u+k_{2} u D_{x}^{\alpha} u+k_{3} D_{t}^{\alpha} D_{x}^{2 \alpha} u=0
$$

Let

$$
u(x, t)=u(\xi), \xi=\frac{a x^{\alpha}}{\Gamma(1+\alpha)}+\frac{b t^{\alpha}}{\Gamma(1+\alpha)}, a \neq 0
$$

then Equation (57) is reduced to the following ordinary differential equation:

$$
b u^{\prime}+k_{1} a u^{\prime}+k_{2} a u u^{\prime}+k_{3} a^{2} b u^{\prime \prime \prime}=0 .
$$

The solution of Equation (57) is in the form of (18), and here, $n=2$ is taken from the homogeneous balance between the highest order derivative $u^{\prime \prime \prime}$ and the nonlinear term $u u^{\prime}$. We obtain the solution of Equation (57) as follows:

$$
u(\xi)=a_{-2} \varphi^{-2}+a_{-1} \varphi^{-1}+a_{0}+a_{1} \varphi+a_{2} \varphi^{2}
$$

Substituting Equation (60) together with its necessary derivatives into Equation (57), the algebraic equation is arranged according to the powers of the function $\varphi^{k}(\xi)$. Then, the following coefficients are obtained:

$$
\begin{aligned}
& \varphi^{-5}:-24 k_{3} a^{2} b a_{-2} \sigma^{3}-2 k_{2} a a_{-2}^{2} \sigma, \\
& \varphi^{-4}:-6 k_{3} a^{2} b a_{-1} \sigma^{3}-3 k_{2} a a_{-1} a_{-2} \sigma \text {, } \\
& \varphi^{-3}:-2 k_{1} a a_{-2} \sigma-2 b a_{-2} \sigma-2 k_{2} a a_{0} a_{-2} \sigma \\
& -k_{2} a a_{-1}^{2} \sigma-2 k_{2} a a_{-2}^{2}-40 k_{3} a^{2} b a_{-2} \sigma^{2} \text {, } \\
& \varphi^{-2}:-k_{1} a a_{-1} \sigma-b a_{-1} \sigma-k_{2} a a_{1} a_{-2} \sigma \\
& -3 k_{2} a a_{-1} a_{-2}-k_{2} a a_{0} a_{-1} \sigma-8 k_{3} a^{2} b a_{-1} \sigma^{2} \text {, } \\
& \varphi^{-1}:-2 b a_{-2}-2 k_{1} a a_{-2}-k_{2} a a_{-1}^{2}-2 k_{2} a a_{0} a_{-2} \\
& -16 k_{3} a^{2} b a_{-2} \sigma \text {, } \\
& \varphi^{0}: k_{1} a a_{1} \sigma-k_{1} a a_{-1}+a_{1} b \sigma-a_{-1} b-k_{2} a a_{1} a_{-2} \\
& +k_{2} a a_{-1} a_{2} \sigma+k_{2} a a_{0} a_{1} \sigma-k_{2} a a_{0} a_{-1} \\
& +2 k_{3} a^{2} b a_{1} \sigma^{2}-2 k_{3} a^{2} b a_{-1} \sigma \text {, } \\
& \varphi^{1}: 2 k_{1} a a_{2} \sigma+2 b a_{2} \sigma+2 k_{2} a a_{0} a_{2} \sigma \\
& +k_{2} a a_{1}^{2} \sigma+16 k_{3} a^{2} b a_{2} \sigma^{2} \text {, } \\
& \varphi^{2}: k_{1} a a_{1}+b a_{1}+k_{2} a a_{2} a_{-1}+k_{2} a a_{1} a_{0} \\
& +3 k_{2} a a_{1} a_{2} \sigma+8 k_{3} a^{2} b a_{1} \sigma \text {, } \\
& \varphi^{3}: 2 k_{1} a a_{2}+2 b a_{2}+2 k_{2} a a_{2}^{2} \sigma+2 k_{2} a a_{0} a_{2} \\
& +k_{2} a a_{1}^{2}+40 k_{3} a^{2} b a_{2} \sigma \text {, } \\
& \varphi^{4}: 6 k_{3} a^{2} b a_{1}+3 k_{2} a a_{1} a_{2} \text {, } \\
& \varphi^{5}: 24 k_{3} a^{2} b a_{2}+2 k_{2} a a_{2}^{2} \text {. }
\end{aligned}
$$

Let the coefficients of $\varphi^{k}(\xi)$ tbe zero. By solving the set of equations given above for $a_{-1}, a_{0}, a_{1}, a, b$, and $\sigma$, we obtain solution sets as follows:

Set 1

$$
\begin{aligned}
a_{-2} & =0, \\
a_{-1} & =0, \\
a_{0} & =a_{0}, \\
a_{1} & =0, \\
a_{2} & =-\frac{12 k_{3} a b}{k_{2}}, \\
a & =a, \\
b & =b, \\
\sigma & =-\frac{k_{1} a+k_{2} a_{0} a+b}{8 k_{3} a^{2} b} .
\end{aligned}
$$

Set 2

$$
a_{-2}=-\frac{3\left(k_{1} a+k_{2} a_{0} a+b\right)^{2}}{16 k_{2} k_{3} a^{3} b},
$$

$a_{-1}=0$,

$$
a_{0}=a_{0} \text {, }
$$




$$
\begin{aligned}
& a_{1}=0, \\
& a_{2}=0, \\
& a=a, \\
& b=b, \\
& \sigma=-\frac{k_{1} a+k_{2} a_{0} a+b}{8 k_{3} a^{2} b} .
\end{aligned}
$$

Set 3

$$
\begin{aligned}
a_{-2} & =-\frac{3\left(k_{1} a+k_{2} a_{0} a+b\right)^{2}}{16 k_{2} k_{3} a^{3} b}, \\
a_{-1} & =0 \\
a_{0} & =a_{0} \\
a_{1} & =0 \\
a_{2} & =-\frac{12 k_{3} a b}{k_{2}} \\
a & =a, \\
b & =b, \\
\sigma & =-\frac{k_{1} a+k_{2} a_{0} a+b}{8 k_{3} a^{2} b} .
\end{aligned}
$$

Thus, we obtain the solution of Equation (57) as $u_{i, j}(\xi)$, $(i=1,2,3 ; j=1,2,3,4,5)$ and $\xi=\left(a x^{\alpha} / \Gamma(1+\alpha)\right)+\left(b t^{\alpha} / \Gamma\right.$ $(1+\alpha)) ; \quad u_{i, j}(\xi)$ is as follows: then

When $\left(k_{1} a+k_{2} a_{0} a+b\right) /\left(8 k_{3} a^{2} b\right)>0$, we have $\sigma<0$,

$$
\begin{aligned}
u_{1,1}(\xi)= & a_{0}-\frac{3\left(k_{1} a+k_{2} a_{0} a+b\right)}{2 k_{2} a} \tanh ^{2} \\
& \cdot\left(\sqrt{\frac{k_{1} a+k_{2} a_{0} a+b}{8 k_{3} a^{2} b} \xi}\right)=u_{2,2}(\xi), \\
u_{2,1}(\xi)= & -\frac{3\left(k_{1} a+k_{2} a_{0} a+b\right)}{2 k_{2} a} \operatorname{coth}^{2} \\
& \cdot\left(\sqrt{\frac{k_{1} a+k_{2} a_{0} a+b}{8 k_{3} a^{2} b}} \xi\right)+a_{0}=u_{1,2}(\xi), \\
u_{3,1}(\xi)= & -\frac{3\left(k_{1} a+k_{2} a_{0} a+b\right)}{2 k_{2} a} \operatorname{coth}^{2}\left(\sqrt{\frac{k_{1} a+k_{2} a_{0} a+b}{8 k_{3} a^{2} b}} \xi\right) \\
& +a_{0}-\frac{3\left(k_{1} a+k_{2} a_{0} a+b\right)}{2 k_{2} a} \tanh ^{2} \\
& \cdot\left(\sqrt{\frac{k_{1} a+k_{2} a_{0} a+b}{8 k_{3} a^{2} b}} \xi\right)=u_{3,2}(\xi) .
\end{aligned}
$$

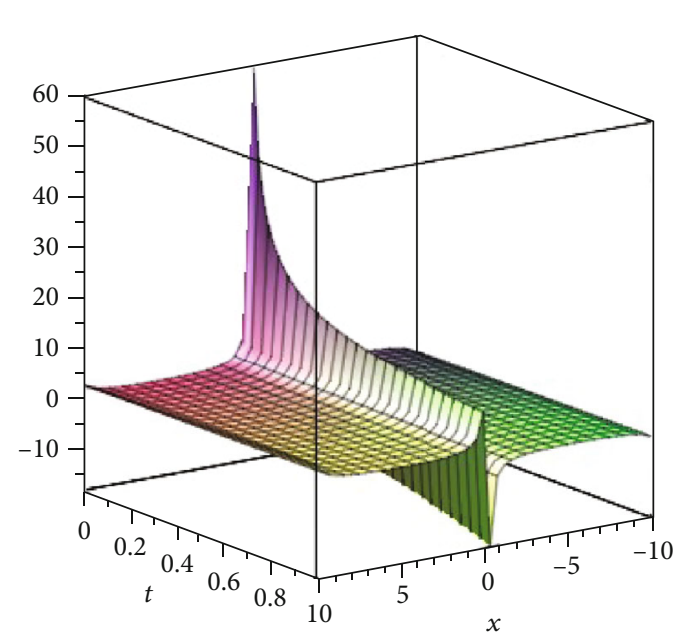

FIgURE 9: $u_{1,3}(x, t)$ of Equation (45).

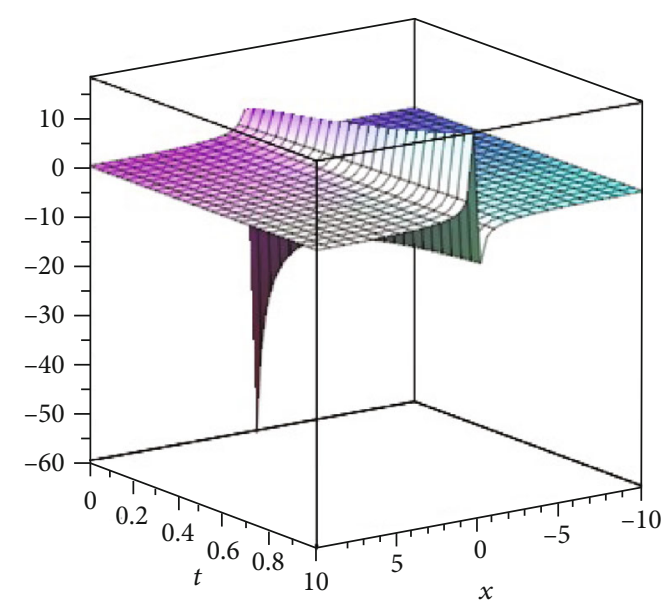

FIgURE 7: $u_{1,1}(x, t)$ of Equation (45).

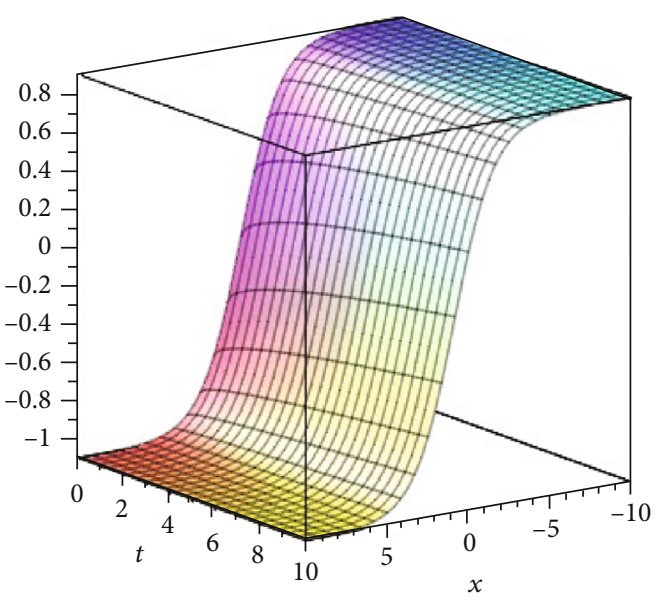

FIgUre 8: $u_{2,1}(x, t)$ of Equation (45). 


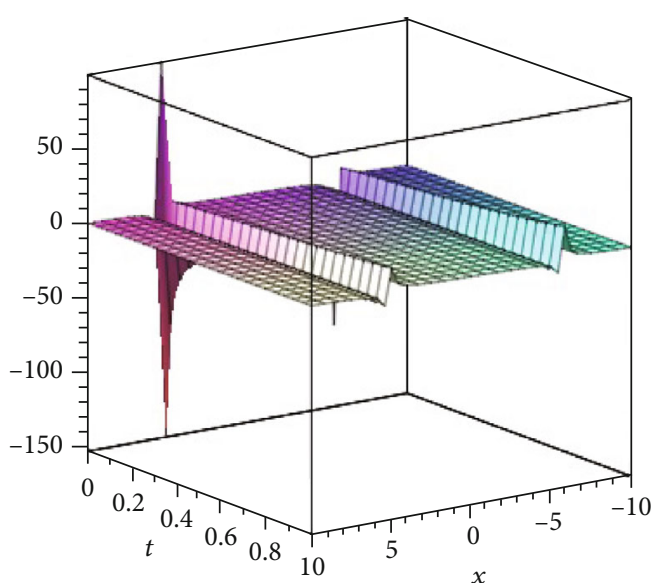

Figure 10: $u_{2,3}(x, t)$ of Equation (45).

When $\left(k_{1} a+k_{2} a_{0} a+b\right) /\left(8 k_{3} a^{2} b\right)<0$, we have $\sigma>0$, then

$$
\begin{aligned}
u_{1,3}(\xi)= & a_{0}+\frac{3\left(k_{1} a+k_{2} a_{0} a+b\right)}{2 k_{2} a} \tan ^{2} \\
& \cdot\left(\sqrt{-\frac{k_{1} a+k_{2} a_{0} a+b}{8 k_{3} a^{2} b} \xi}\right)=u_{2,4}(\xi), \\
u_{2,3}(\xi)= & \frac{3\left(k_{1} a+k_{2} a_{0} a+b\right)}{2 k_{2} a} \cot ^{2}\left(\sqrt{-\frac{k_{1} a+k_{2} a_{0} a+b}{8 k_{3} a^{2} b} \xi}\right) \\
& +a_{0}=u_{1,4}(\xi), \\
u_{3,3}(\xi)= & \frac{3\left(k_{1} a+k_{2} a_{0} a+b\right)}{2 k_{2} a} \cot ^{2}\left(\sqrt{-\frac{k_{1} a+k_{2} a_{0} a+b}{8 k_{3} a^{2} b}} \xi\right) \\
& +a_{0}+\frac{3\left(k_{1} a+k_{2} a_{0} a+b\right)}{2 k_{2} a} \tan ^{2} \\
& \cdot\left(\sqrt{-\frac{k_{1} a+k_{2} a_{0} a+b}{8 k_{3} a^{2} b}} \xi\right)=u_{3,4}(\xi) .
\end{aligned}
$$

When $\left(k_{1} a+k_{2} a_{0} a+b\right) /\left(8 k_{3} a^{2} b\right)=0, a_{-2}=0$, we have $\sigma=0$, then

$$
u_{1,5}(\xi)=a_{0}-\frac{12 k_{3} a b \Gamma^{2}(1+\alpha)}{k_{2}\left(\xi^{\alpha}+\omega\right)^{2}}=u_{3,5}(\xi)
$$

Solutions $u_{i, 1}$ and $u_{i, 2}(i=1,2,3)$ describe the multiple soliton. Solutions $u_{i, 3}$ and $u_{i, 4}(i=1,2,3)$ represent the exact periodic traveling wave solutions.

Figures 11-14 present the solutions: $u_{1,1}, u_{2,1}, u_{2,3}$, and $u_{1,5}$ of the generalized compound $\mathrm{KdV}$-Burgers equation with $0<x<1,0<t<2$. Solution $u_{1,1}$ and solution $u_{2,1}$ are presented for values $\alpha=0.5, k_{1}=k_{2}=1, k_{3}=k_{4}=-1, a=-4$, $b=1, a_{0}=0$, and $y=0 ; u_{2,3}$ is presented for values $\alpha=0.5$, $k_{1}=k_{2}=1, k_{3}=k_{4}=-1, a=4, b=1, a_{0}=0$, and $y=0$; solu-

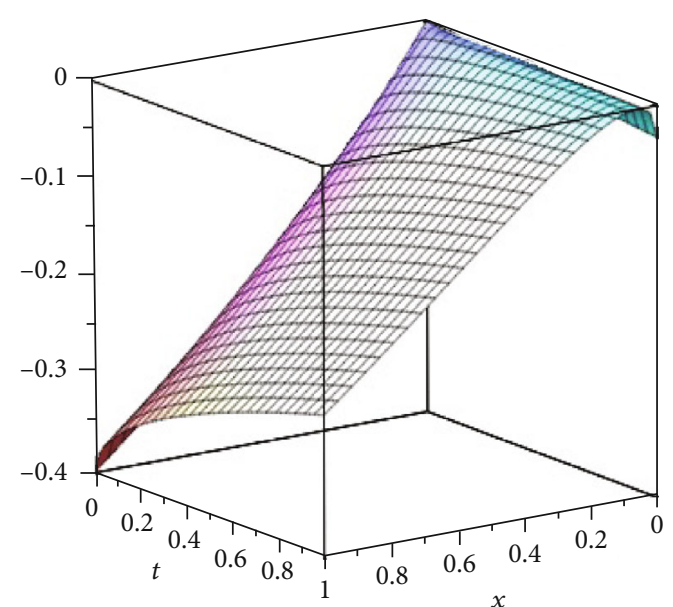

Figure 11: $u_{1,1}(x, t)$ of Equation (57).

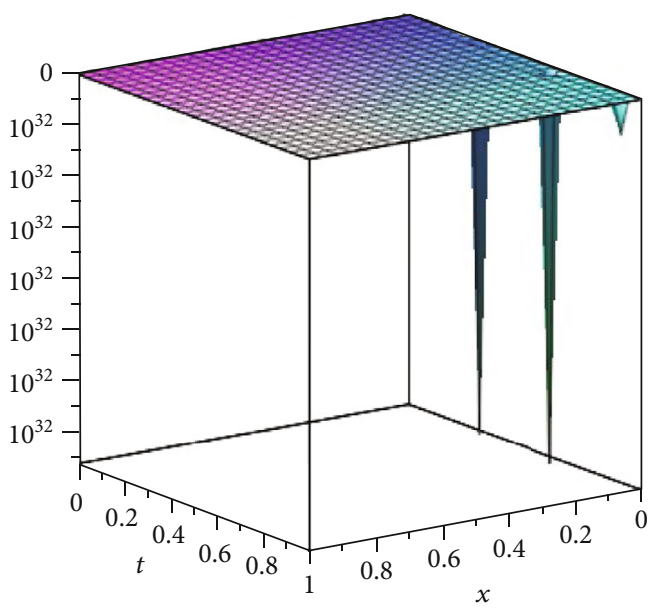

FIGURE 12: $u_{2,1}(x, t)$ of Equation (57).

tion $u_{1,5}$ is presented for values $\alpha=0.5, k_{1}=k_{2}=1, k_{3}=k_{4}=$ $-1, a=4, b=-4, a_{0}=0, \omega=0$, and $y=0$.

3.4. The $(3+1)$-Space-Time Fractional Zakharov-Kuznetsov Equation. The $(3+1)$-space-time fractional ZakharovKuznetsov Equation is given by

$$
D_{t}^{\alpha} u+k_{1} u D_{x}^{\alpha} u+k_{2} D_{x}^{3 \alpha} u+k_{3} D_{x}^{\alpha} D_{y}^{2 \alpha} u+k_{4} D_{x}^{\alpha} D_{z}^{2 \alpha} u=0,
$$

Let

$$
\begin{aligned}
u(x, t)= & u(\xi), \xi=\frac{a t^{\alpha}}{\Gamma(1+\alpha)}+\frac{b x^{\alpha}}{\Gamma(1+\alpha)}+\frac{c y^{\alpha}}{\Gamma(1+\alpha)} \\
& +\frac{d z^{\alpha}}{\Gamma(1+\alpha)}, a \neq 0
\end{aligned}
$$

then Equation (69) is reduced to the ordinary differential equation as

$$
a u^{\prime}+k_{1} b u u^{\prime}+k_{2} b^{3} u^{\prime \prime \prime}+k_{3} b c^{2} u^{\prime \prime \prime}+k_{4} b d^{2} u^{\prime \prime \prime}=0 .
$$




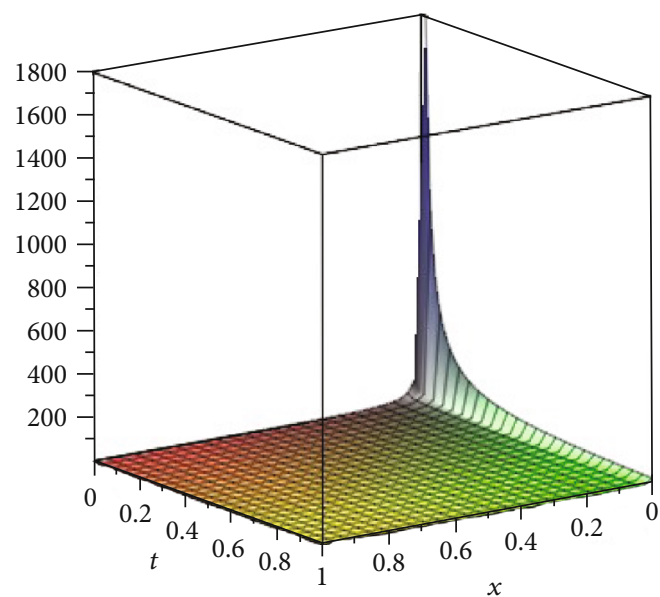

Figure 13: $u_{2,3}(x, t)$ of Equation (57).

The solution of Equation (71) is the form (18), and here, $n=2$ is taken from the homogeneous balance between the highest order derivative $u^{\prime \prime \prime}$ and the nonlinear term $u u^{\prime}$. We obtain the solution of Equation (71) as

$$
u(\xi)=a_{-2} \varphi^{-2}+a_{-1} \varphi^{-1}+a_{0}+a_{1} \varphi+a_{2} \varphi^{2}
$$

Substituting Equation (72) together with its necessary derivatives into Equation (71), the algebraic equation is arranged according to the powers of the function $\varphi^{k}(\xi)$. Then, the following coefficients are obtained:

$$
\begin{aligned}
\varphi^{-5}: & -2 k_{1} b a_{-2}^{2} \sigma+24 \lambda a_{-2} \sigma^{3}, \\
\varphi^{-4}: & -3 k_{1} b a_{-1} a_{-2} \sigma+6 \lambda a_{-1} \sigma^{3}, \\
\varphi^{-3}: & -2 a a_{-2} \sigma-2 k_{1} b a_{0} a_{-2} \sigma-k_{1} b a_{-1}^{2} \sigma \\
& -2 k_{1} b a_{-2}^{2}+40 \lambda a_{-2} \sigma^{2}, \\
\varphi^{-2}: & -a a_{-1} \sigma-3 k_{1} b a_{-1} a_{-2}-k_{1} b a_{0} a_{-1} \sigma \\
& -k_{1} b a_{1} a_{-2} \sigma+8 \lambda a_{-1} \sigma^{2}, \\
\varphi^{-1}: & -2 a a_{-2}-k_{1} b a_{-1}^{2}-2 k_{1} b a_{0} a_{-2}+16 \lambda a_{-2} \sigma, \\
\varphi^{0}: & a a_{1} \sigma-a a_{-1}-k_{1} b a_{-2} a_{1}+k_{1} b a_{-1} a_{2} \sigma \\
& +k_{1} b a_{0} a_{1} \sigma-k_{1} b a_{0} a_{-1}-2 \lambda a_{1} \sigma^{2}+2 \lambda a_{-1} \sigma \\
\varphi^{1}: & 2 a a_{2} \sigma+2 k_{1} b a_{0} a_{2} \sigma+k_{1} b a_{1}^{2} \sigma-16 \lambda a_{2} \sigma^{2}, \\
\varphi^{2}: & a a_{1}+k_{1} b a_{-1} a_{2}+k_{1} b a_{0} a_{1}+3 k_{1} b a_{1} a_{2} \sigma-8 \lambda a_{1} \sigma, \\
\varphi^{3}: & 2 a a_{2}+2 k_{1} b a_{2}^{2} \sigma+2 k_{1} b a_{0} a_{2}+k_{1} b a_{1}^{2}-40 \lambda a_{2} \sigma \\
\varphi^{4}: & 3 k_{1} b a_{1} a_{2}-6 \lambda a_{1}, \\
\varphi^{5}: & 2 k_{1} b a_{2}^{2}-24 \lambda a_{2} .
\end{aligned}
$$

where $\lambda=-k_{2} b^{3}-k_{3} b c^{2}-k_{4} b d^{2}$.

Let the coefficients of $\varphi^{k}(\xi)$ be zero. By solving the set of equations given above for $a_{-1}, a_{0}, a_{1}, a, b$, and $\sigma$, we obtain solution sets as follows:

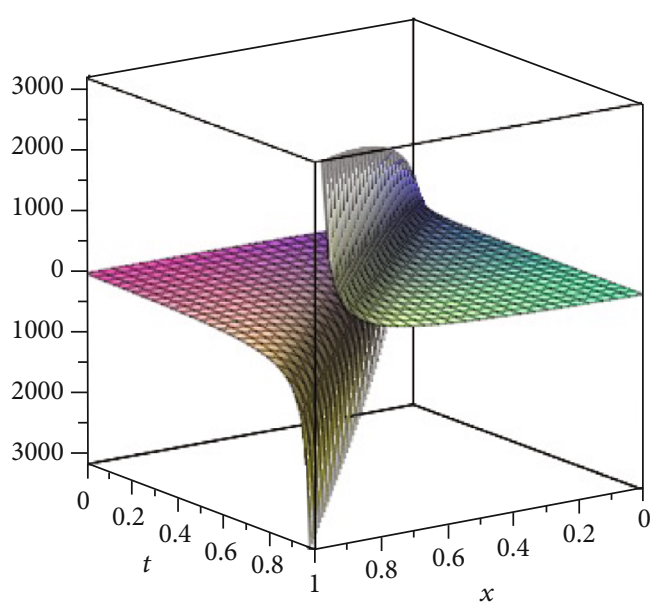

Figure 14: $u_{1,5}(x, t)$ of Equation (57).

Set 1

$$
\begin{aligned}
a_{-2} & =0, \\
a_{-1} & =0, \\
a_{0} & =a_{0}, \\
a_{1} & =0, \\
a_{2} & =\frac{12 \lambda}{k_{1}}, \\
a & =a, \\
b & =b, \\
c & =c, \\
d & =d, \\
\sigma & =\frac{a+k_{1} a_{0} b}{8 b \lambda} .
\end{aligned}
$$

Set 2

$$
\begin{aligned}
a_{-2} & =\frac{3\left(a+k_{1} a_{0} b\right)^{2}}{16 k_{1} b^{2} \lambda}, \\
a_{-1} & =0 \\
a_{0} & =a_{0}, \\
a_{0} & =a_{0}, \\
a_{1} & =0 \\
a_{2} & =0 \\
a & =a \\
b & =b \\
c & =c \\
d & =d, \\
\sigma & =\frac{a+k_{1} a_{0} b}{8 b \lambda} .
\end{aligned}
$$


Set 3

$$
\begin{aligned}
a_{-2} & =\frac{3\left(a+k_{1} a_{0} b\right)^{2}}{16 k_{1} b^{2} \lambda}, \\
a_{-1} & =0, \\
a_{0} & =a_{0}, \\
a_{1} & =0, \\
a_{2} & =\frac{12 \lambda}{k_{1}}, \\
a & =a, \\
b & =b, \\
c & =c, \\
d & =d, \\
\sigma & =\frac{a+k_{1} a_{0} b}{8 b \lambda} .
\end{aligned}
$$

Thus, we obtain the solution of Equation (59) as $u_{i, j}(\xi)$, $(i=1,2,3 ; j=1,2,3,4,5)$ and $\xi=\left(a t^{\alpha} / \Gamma(1+\alpha)\right)+\left(b x^{\alpha} / \Gamma(1\right.$ $+\alpha))+\left(c y^{\alpha} / \Gamma(1+\alpha)\right)+\left(d z^{\alpha} / \Gamma(1+\alpha)\right) ; u_{i, j}(\xi)$ is tas follows:

When $\left(a+k_{1} a_{0} b\right) /(8 b \lambda)<0$, we have $\sigma<0$, then

$$
\begin{aligned}
u_{1,1}(\xi)= & a_{0}-\frac{3\left(a+k_{1} a_{0} b\right)}{2 k_{1} b} \tanh ^{2}\left(\sqrt{-\frac{a+k_{1} a_{0} b}{8 b \lambda} \xi}\right), \\
u_{2,1}(\xi)= & -\frac{12 \lambda\left(a+k_{1} a_{0} b\right)}{k_{1}} \operatorname{coth}^{2}\left(\sqrt{-\frac{a+k_{1} a_{0} b}{8 b \lambda} \xi}\right)+a_{0}, \\
u_{3,1}(\xi)= & -\frac{12 \lambda\left(a+k_{1} a_{0} b\right)}{k_{1}} \operatorname{coth}^{2}\left(\sqrt{-\frac{a+k_{1} a_{0} b}{8 b \lambda} \xi}\right) \\
& +a_{0}-\frac{3\left(a+k_{1} a_{0} b\right)}{2 k_{1} b} \tanh ^{2}\left(\sqrt{-\frac{a+k_{1} a_{0} b}{8 b \lambda} \xi}\right), \\
u_{1,2}(\xi)= & a_{0}-\frac{3\left(a+k_{1} a_{0} b\right)}{2 k_{1} b} \operatorname{coth}^{2}\left(\sqrt{-\frac{a+k_{1} a_{0} b}{8 b \lambda} \xi}\right), \\
u_{2,2}(\xi)= & -\frac{12 \lambda\left(a+k_{1} a_{0} b\right)}{k_{1}} \tanh ^{2}\left(\sqrt{-\frac{a+k_{1} a_{0} b}{8 b \lambda} \xi}\right)+a_{0}, \\
u_{3,2}(\xi)= & -\frac{12 \lambda\left(a+k_{1} a_{0} b\right)}{k_{1}} \tanh ^{2}\left(\sqrt{-\frac{a+k_{1} a_{0} b}{8 b \lambda}} \xi\right) \\
& +a_{0}-\frac{3\left(a+k_{1} a_{0} b\right)}{2 k_{1} b} \operatorname{coth}^{2}\left(\sqrt{-\frac{a+k_{1} a_{0} b}{8 b \lambda}} \xi\right) .
\end{aligned}
$$

When $\left(a+k_{1} a_{0} b\right) /(8 b \lambda)>0$, we have $\sigma>0$, then

$$
u_{1,3}(\xi)=a_{0}+\frac{3\left(a+k_{1} a_{0} b\right)}{2 k_{1} b} \tan ^{2}\left(\sqrt{\frac{a+k_{1} a_{0} b}{8 b \lambda}} \xi\right),
$$

$$
\begin{aligned}
u_{2,3}(\xi)= & \frac{12 \lambda\left(a+k_{1} a_{0} b\right)}{k_{1}} \cot ^{2}\left(\sqrt{\frac{a+k_{1} a_{0} b}{8 b \lambda} \xi}\right)+a_{0}, \\
u_{3,3}(\xi)= & \frac{12 \lambda\left(a+k_{1} a_{0} b\right)}{k_{1}} \cot ^{2}\left(\sqrt{\frac{a+k_{1} a_{0} b}{8 b \lambda} \xi}\right) \\
& +a_{0}+\frac{3\left(a+k_{1} a_{0} b\right)}{2 k_{1} b} \tan ^{2}\left(\sqrt{\frac{a+k_{1} a_{0} b}{8 b \lambda}} \xi\right), \\
u_{1,4}(\xi)= & a_{0}+\frac{3\left(a+k_{1} a_{0} b\right)}{2 k_{1} b} \cot ^{2}\left(\sqrt{\frac{a+k_{1} a_{0} b}{8 b \lambda} \xi}\right), \\
u_{2,4}(\xi)= & \frac{12 \lambda\left(a+k_{1} a_{0} b\right)}{k_{1}} \tan ^{2}\left(\sqrt{\frac{a+k_{1} a_{0} b}{8 b \lambda} \xi}\right)+a_{0}, \\
u_{3,4}(\xi)= & \frac{12 \lambda\left(a+k_{1} a_{0} b\right)}{k_{1}} \tan ^{2}\left(\sqrt{\frac{a+k_{1} a_{0} b}{8 b \lambda} \xi}\right) \\
& +a_{0}+\frac{3\left(a+k_{1} a_{0} b\right)}{2 k_{1} b} \cot ^{2}\left(\sqrt{\frac{a+k_{1} a_{0} b}{8 b \lambda}} \xi\right) .
\end{aligned}
$$

When $\left(a+k_{1} a_{0} b\right) /(8 b \lambda)=0$, we have $\sigma=0$, then

$$
u_{1,5}(\xi)=a_{0}+\frac{12 \lambda \Gamma^{2}(1+\alpha)}{k_{1}\left(\xi^{\alpha}+\omega\right)^{2}}=u_{3,5}(\xi) .
$$

Solutions $u_{i, 1}$ and $u_{i, 2}(i=1,2,3)$ describe the multiple soliton. Solutions $u_{i, 3}$ and $u_{i, 4}(i=1,2,3)$ represent the exact periodic traveling wave solutions.

Figures $15-18$ present the solutions: $u_{1,1}, u_{1,3}, u_{2,3}$, and $u_{1,5}$ of the generalized compound KdV-Burgers equation with $0<x<1,0<t<2$. Solution $u_{1,1}$ is presented for values $\alpha=0.5, k_{1}=k_{2}=1, k_{3}=k_{4}=-1, a=-4, b=1, c=1$, $d=1, a_{0}=0$, and $y=z=0$; solution $u_{1,3}$ and $u_{2,3}$ are presented for values $\alpha=0.5, k_{1}=k_{2}=1, k_{3}=k_{4}=-1, a=4, b$ $=1, c=1, d=1, a_{0}=0$, and $y=z=0$; solution $u_{1,5}$ is presented for values $\alpha=0.5, k_{1}=k_{2}=1, k_{3}=k_{4}=-1, a=-4$, $b=1, c=1, d=1, a_{0}=4, \omega=0$, and $y=z=0$.

\section{Results and Discussion}

The generalized time fractional biological population model, the generalized time fractional compound KdV-Burgers equation, the space-time fractional regularized long-wave equation, and the $(3+1)$-space-time fractional ZakharovKuznetsov equation have gained the focus of many studies due to their frequent appearance in various applications.

The improved fractional subequation method has several advantages according to other traditional methods. Applying a suitable fractional complex transform $u\left(t, x_{1}, x_{2}, \cdots, x_{m}\right)=$ $u(\xi), \xi=\xi\left(t, x_{1}, x_{2}, \cdots, x_{m}\right)$ and chain rule, the nonlinear fractional differential equations with the modified RiemannLiouville derivative are converted into the nonlinear ordinary differential equations. This is a significant impact because 


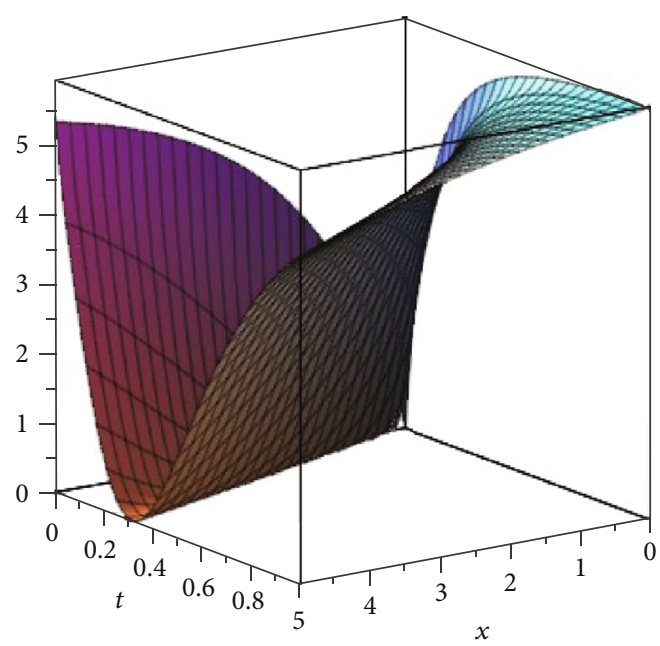

Figure 15: $u_{1,1}(x, 0,0, t)$ of Equation (69).

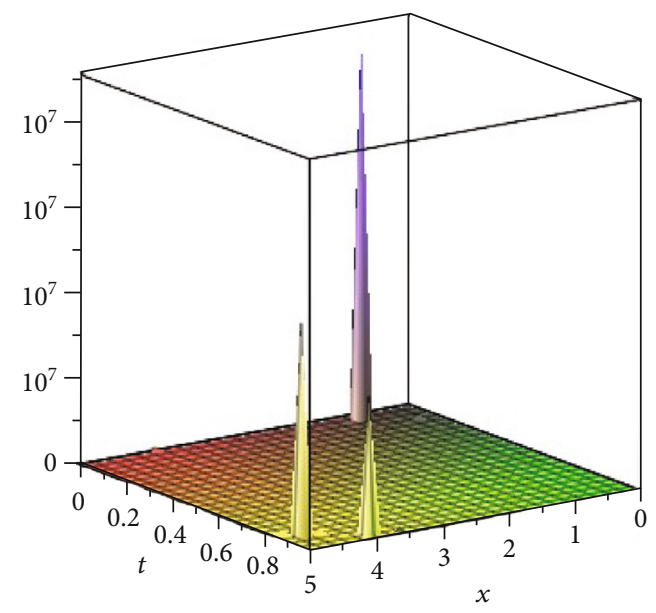

Figure 16: $u_{1,3}(x, 0,0, t)$ of Equation (69).

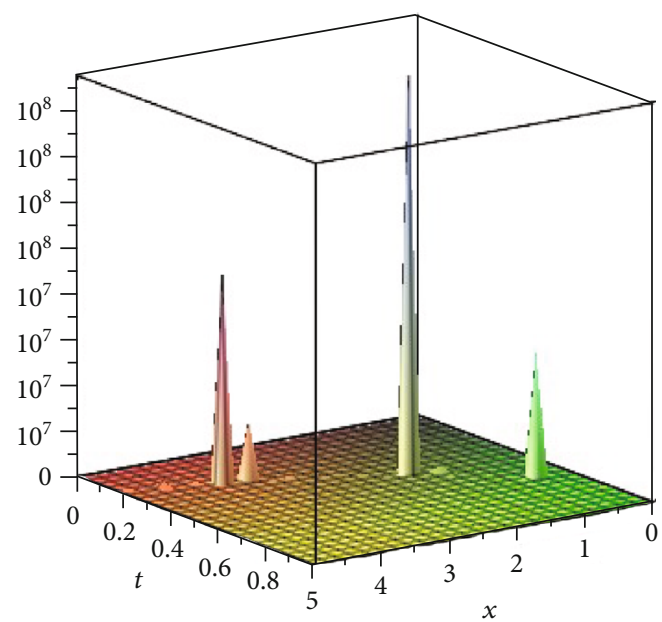

Figure 17: $u_{2,3}(x, 0,0, t)$ of Equation (69).

neither Caputo definition nor Riemann-Liouville definition satisfies the chain rule. With the help of the Riccati equation, the method has been employed for finding the exact analytical solutions of these equations.

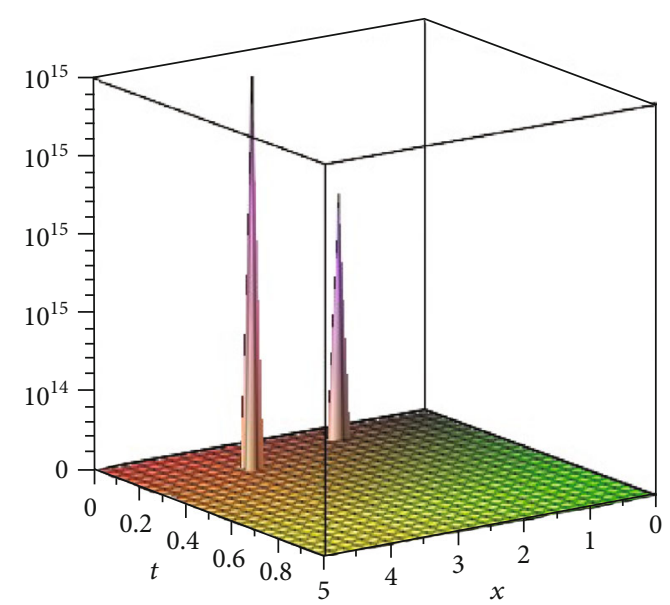

FIgURE 18: $u_{1,5}(x, 0,0, t)$ of Equation (69).

These obtained solutions are traveling solutions. Furthermore, solutions $u_{i, 1}(\xi)$ and $u_{i, 2}(\xi)$ describe the solitons which are everywhere in nature. $u_{i, 3}(\xi)$ and $u_{i, 4}(\xi)$ represent the exact periodic traveling wave solutions.

The improved fractional subequation method is reliable and effective for finding more solutions for some spacetime fractional nonlinear differential equations. We can substitute $\varphi^{\prime}=r+p \varphi+q \varphi^{2}$ [39] for $\varphi^{\prime}=\sigma+\varphi^{2}$, then, we will obtain more solutions.

\section{Data Availability}

All data included in this study are available upon request by contact with the corresponding author.

\section{Conflicts of Interest}

The authors declare that they have no conflicts of interest.

\section{Acknowledgments}

This research is supported by the Open Project of State Key Laboratory of Environment-Friendly Energy Materials (19kfhg08), the Natural Science Foundation (61473338), and Hubei Province Key Laboratory of Systems Science in Metallurgical Process (Y201705).

\section{References}

[1] I. Podlubny, Fractional Differential Equations, Academic Press, San Diego, 1999.

[2] B. J. West, M. Bolognab, and P. Grigolini, Physics of Fractal Operators, Springer, New York, 2003.

[3] K. S. Miller and B. Ross, An Introduction to the Fractional Calculus and Fractional Differential Equations, Wiley, New York, 1993.

[4] S. G. Samko, A. A. Kilbas, and O. I. Marichev, Fractional Integrals and Derivatives: Theory and Applications, Gordon and Breach, Y verdon, 1993.

[5] A. A. Kilbas, H. M. Srivastava, and J. J. Trujillo, Theory and Applications of Fractional Differential Equations, Elsevier Science, Amsterdam, 2006. 
[6] X. Zhang, L. Liu, and Y. Wu, "Multiple positive solutions of a singular fractional differential equation with negatively perturbed term," Mathematical and Computer Modelling, vol. 55, no. 3-4, pp. 1263-1274, 2012.

[7] J. He, X. Zhang, L. Liu, Y. Wu, and Y. Cui, "A singular fractional Kelvin-Voigt model involving a nonlinear operator and their convergence properties," Boundary Value Problems, vol. 2019, no. 1, 2019.

[8] C. H. Gu, H. S. Hu, and Z. X. Zhou, Darboux Transformations in Soliton Theory and its Geometric Applications, Shanghai Scientific and Technical Publishers, Shanghai, 1999.

[9] T. Xia, H. Zhang, and Z. Yan, "New explicit and exact travelling wave solutions for a class of nonlinear evolution equations," Applied Mathematics and Mechanics, vol. 22, no. 7, pp. 788-793, 2001.

[10] N. H. Sweilam, M. M. Khader, and R. F. Al-Bar, "Numerical studies for a multi-order fractional differential equation," Physics Letters A, vol. 371, no. 1-2, pp. 26-33, 2007.

[11] M. Inc, "The approximate and exact solutions of the spaceand time-fractional Burgers equations with initial conditions by variational iteration method," Journal of Mathematical Analysis and Applications, vol. 345, no. 1, pp. 476-484, 2008.

[12] Q. Huang, G. Huang, and H. Zhan, "A finite element solution for the fractional advection-dispersion equation," Advances in Water Resources, vol. 31, no. 12, pp. 1578-1589, 2008.

[13] A. Esen, Y. Ucar, N. Yagmurlu, and O. Tasbozan, "A Galerkin finite element method to solve fractional diffusion and fractional diffusion-wave equations," Mathematical Modelling and Analysis, vol. 18, no. 2, pp. 260-273, 2013.

[14] R. Kumar, R. S. Kaushal, and A. Prasad, "Some new solitary and travelling wave solutions of certain nonlinear diffusionreaction equations using auxiliary equation method," Physics Letters A, vol. 372, no. 19, pp. 3395-3399, 2008.

[15] M. Cui, "Compact finite difference method for the fractional diffusion equation," Journal of Computational Physics, vol. 228, no. 20, pp. 7792-7804, 2009.

[16] Y. Zhang, "A finite difference method for fractional partial differential equation," Applied Mathematics and Computation, vol. 215, no. 2, pp. 524-529, 2009.

[17] A. M. A. El-Sayed and M. Gaber, "The Adomian decomposition method for solving partial differential equations of fractal order in finite domains," Physics Letters A, vol. 359, no. 3, pp. 175-182, 2006.

[18] M. Safari, D. D. Ganji, and M. Moslemi, "Application of He's variational iteration method and Adomian's decomposition method to the fractional KdV-Burgers-Kuramoto equation," Computers \& Mathematcs with Applications, vol. 58, no. 1112, pp. 2091-2097, 2009.

[19] R. Kumar, R. S. Kaushal, and A. Prasad, "Solitary wave solutions of selective nonlinear diffusion-reaction equations using homogeneous balance method," Pramana-Journal of Physics, vol. 75, no. 4, pp. 607-616, 2010.

[20] J. M. Zuo and Y. M. Zhang, "The Hirota bilinear method for the coupled Burgers equation and the high-order BoussinesqBurgers equation," Chinese Physics B, vol. 20, no. 1, article 010205, 2011.

[21] L. Song and H. Zhang, "Solving the fractional BBM-Burgers equation using the homotopy analysis method," Chaos, Solitons \& Fractals, vol. 40, no. 4, pp. 1616-1622, 2009.

[22] A. A. M. Arafa, S. Z. Rida, and H. Mohamed, "Homotopy analysis method for solving biological population model," Com- munications in Theoretical Physics, vol. 56, no. 5, pp. 797800, 2011.

[23] A. Bekir and O. Guner, "Exact solutions of nonlinear fractional differential equations by $\left(G^{\prime} / G\right)$-expansion method," Chinese Physics B, vol. 22, no. 11, article 110202, 2013.

[24] M. H. Uddin, M. A. Akbar, M. A. Khan, and M. A. Haque, "Close form solutions of the fractional generalized reaction duffing model and the density dependent fractional diffusion reaction equation," Applied and Computational Mathematics, vol. 6, no. 4, pp. 177-184, 2017.

[25] B. Tang, Y. He, L. Wei, and X. Zhang, “A generalized fractional sub-equation method for fractional differential equations with variable coefficients," Physics Letters A, vol. 376, no. 38-39, pp. 2588-2590, 2012.

[26] H. Jafari, H. Tajadodi, D. Baleanu, A. al-Zahrani, Y. Alhamed, and A. Zahid, "Fractional sub-equation method for the fractional generalized reaction Duffing model and nonlinear fractional Sharma-Tasso-Olver equation," Open Physics, vol. 11, no. 10, pp. 1482-1486, 2013.

[27] M. Eslami, B. Fathi Vajargah, M. Mirzazadeh, and A. Biswas, "Application of first integral method to fractional partial differential equations," Indian Journal of Physics, vol. 88, no. 2, pp. 177-184, 2014.

[28] B. Lu, "The first integral method for some time fractional differential equations," Journal of Mathematical Analysis and Applications, vol. 395, no. 2, pp. 684-693, 2012.

[29] G. Wang and T. Xu, "The improved fractional sub-equation method and its applications to nonlinear fractional equations," Theoretical and Mathematical Physics, vol. 66, no. 3, pp. 595602, 2014.

[30] S. Sahoo and S. Saha Ray, "Improved fractional sub-equation method for $(3+1)$-dimensional generalized fractional KdVZakharov-Kuznetsov equations," Computers and Mathematics with Applications, vol. 70, no. 2, pp. 158-166, 2015.

[31] A. Sonmezoglu, "Exact solutions for some fractional differential equations," Advances in Mathematical Physics, vol. 2015, Article ID 567842, 10 pages, 2015.

[32] M. Kaplan, A. Bekir, and A. Akbulut, "A generalized Kudryashov method to some nonlinear evolution equations in mathematical physics," Nonlinear Dynamics, vol. 85, no. 4, pp. 2843-2850, 2016.

[33] S. T. Mohyud-Din and S. Bibi, "Exact solutions for nonlinear fractional differential equations using exponential rational function method," Optical and Quantum Electronics, vol. 49, no. 2, 2017.

[34] S. Zhang, Q. A. Zong, D. Liu, and Q. Gao, "A generalized expfunction method for fractional Riccati differential equations," Communications in Fractional Calculus, vol. 1, pp. 48-52, 2010.

[35] A. Bekir, O. Guner, and A. C. Cevikel, "Fractional complex transform and exp-function methods for fractional differential equations," Abstract and Applied Analysis, vol. 2013, 8 pages, 2013.

[36] A. R. Adem, "The generalized (1+1)-dimensional and (2+1)dimensional Ito equations: Multiple exp-function algorithm and multiple wave solutions," Computers \& Mathematcs with Applications, vol. 71, no. 6, pp. 1248-1258, 2016.

[37] D. Lu, A. Seadawy, and M. Arshad, “Applications of extended simple equation method on unstable nonlinear Schrödinger equations," Optik, vol. 140, pp. 136-144, 2017. 
[38] G. Jumarie, "Modified Riemann-Liouville derivative and fractional Taylor series of nondifferentiable functions further results," Computers and Mathematics with Applications, vol. 51, no. 9-10, pp. 1367-1376, 2006.

[39] J. Manafian and M. Lakestani, "A new analytical approach to solve some of the fractional-order partial differential equations," Indian Journal of Physics, vol. 91, no. 3, pp. 243-258, 2017.

[40] A. Kurt, Y. Cenesiz, and O. Tasbozan, "On the Solution of Burgers' Equation with the New Fractional Derivative," Open Physics, vol. 13, no. 1, pp. 355-360, 2015.

[41] E. A. B. Abdel-Salam and G. F. Hassan, "Solutions to class of linear and nonlinear fractional differential equations," Communications in Theoretical Physics, vol. 65, no. 2, pp. 127135, 2016.

[42] B. Karaagac and Department of Mathematic Education, Adıyaman University, Adıyaman, Turkey, "New exact solutions for some fractional order differential equations via improved sub-equation method," Discrete \& Continuous Dynamical Systems - S, vol. 12, no. 3, pp. 447-454, 2019. 